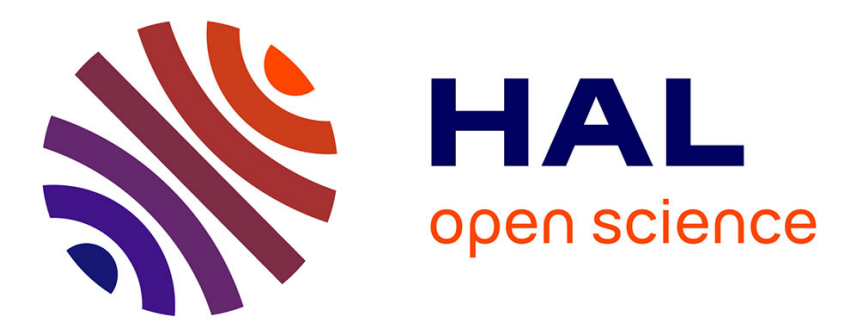

\title{
Basal ganglia oscillations: the role of delays and external excitatory nuclei
}

\author{
Ihab Haidar, William Pasillas-Lépine, Elena Panteley, Antoine Chaillet
}

\section{To cite this version:}

Ihab Haidar, William Pasillas-Lépine, Elena Panteley, Antoine Chaillet. Basal ganglia oscillations: the role of delays and external excitatory nuclei. 12th European Control Conference (ECC 2013), Jul 2013, Zurich, Switzerland. pp.1-6, 10.23919/ecc.2013.6669566 . hal-00742100v3

\section{HAL Id: hal-00742100 \\ https://hal-centralesupelec.archives-ouvertes.fr/hal-00742100v3}

Submitted on 19 Nov 2012

HAL is a multi-disciplinary open access archive for the deposit and dissemination of scientific research documents, whether they are published or not. The documents may come from teaching and research institutions in France or abroad, or from public or private research centers.
L'archive ouverte pluridisciplinaire HAL, est destinée au dépôt et à la diffusion de documents scientifiques de niveau recherche, publiés ou non, émanant des établissements d'enseignement et de recherche français ou étrangers, des laboratoires publics ou privés. 


\title{
Basal ganglia oscillations: the role of delays and external excitatory nuclei
}

\author{
${ }^{* \dagger}$ Ihab Haidar, ${ }^{\ddagger}$ William Pasillas-Lépine, ${ }^{\S}$ Elena Panteley, and ${ }^{\top}$ Antoine Chaillet $\|$
}

November 18, 2012

\begin{abstract}
Basal ganglia are interconnected deep brain structures involved in movement generation. Their oscillations in the beta band $(13-30 \mathrm{~Hz})$ is known to be linked to Parkinson's disease motor symptoms. In this paper, we provide conditions under which these oscillations may occur, by explicitly considering the role of the pedunculopontine nucleus (PPN). We analyze the existence of equilibria in the associated firing-rates dynamics, and study their stability by relying on a delayed MIMO frequency analysis. The results are illustrated by simulations.
\end{abstract}

\section{Introduction}

Basal ganglia are deep brain structures involved in voluntary motor control as well as cognitive and motivational processes $[8,17]$. They have been studied extensively in connection with a variety of pathological observations such as Parkinson's disease [23]. Some evidence suggests that the advance of parkinsonism is highly correlated to the presence of abnormal oscillations in the beta band $(13-30 \mathrm{~Hz})$ within the basal ganglia [3]. There exist several hypothesis about the origin of these oscillations. Some of them emphasize the cortical [25] or striatal [16] origin of the phenomenon. But another popular assumption is that these oscillations may originate from the system composed of two excitatory-inhibitory basal ganglia nuclei: the subthalamic nucleus (STN), which is an excitatory nucleus, and the globus pallidus pars externa (GPe), which is an inhibitory nucleus [20]. Since basal ganglia are highly interconnected with the pedunculopontine nucleus (PPN) $[4,9,21]$, this nucleus might have an influence on their oscillatory activity. The aim of our paper is to quantify this influence, with the help of control theory tools.

To explore the origin of pathological oscillations in the basal ganglia, several works relying on firingrate models have been proposed $[10,13,12,19]$. These models are formulated in terms of an ordinary differential equation that rule the evolution of the number of spikes per time-unit within the considered neuronal population [6]. Other works $[22,11]$ exploit a microscopic approach in which every neuronal cell is modeled individually. In [10], the authors exploit a spike-rate model of the STN and GPe populations to derive analytical conditions under which beta oscillations occur. These conditions have been improved in $[18,19]$ to provide tighter conditions for the existence of oscillations. The particular role of PPN within the basal ganglia has been specifically addressed in [15], where the authors study how the PPN responds to physiological and pathological inputs of the basal ganglia.

Here we develop a mathematical model that describes the interaction between three neuronal populations: PPN, STN and GPe (see Section 2). To analyze this model, we extend the approach developed in [18] for two nuclei only. We first study the existence and uniqueness of equilibrium points (see Section 3). We derive necessary and sufficient conditions for the existence of multiple equilibria. Additionally, we propose a sufficient condition for global asymptotic stability in the absence of delays. Then, the

\footnotetext{
${ }^{*}$ Corresponding author.

${ }^{\dagger}$ Ihab Haidar is with L2S-Supélec, 3, rue Joliot-Curie, 91192, Gif-sur-Yvette, France, ihab.haidar@lss.supelec.fr

${ }^{\ddagger}$ William Pasillas-Lépine is with CNRS-L2S-Supélec, same address, pasillas@lss.supelec.fr

$\S$ Elena Panteley is with CNRS-L2S-Supélec, same address, panteley@lss.supelec.fr

ฯAntoine Chaillet is with Univ. Paris-Sud 11-L2S-Supélec, same address, antoine.chaillet@lss.supelec.fr

$\|$ The research leading to these results has received funding from the European Union Seventh Framework Programme [FP7/2007-2013] under grant agreement n257462 HYCON2 Network of excellence.
} 
system is linearized and the MIMO Nyquist stability criterion [5] is applied to the feedback loop of the linearized system (see Section 4) to derive explicit conditions on the delays and interconnection gains for the asymptotic stability of the network, and hence the absence of pathological oscillations. The proofs are postponed to the section 6 , for lightening the presentation. These results are illustrated by numerical simulations (see Section 5).

\section{Model description}

As we mentionned in the introduction, our objective is to analyze pathological oscillations in the basal ganglia. To characterize the firing rate of neural populations in STN, GPe and PPN, we use the well described firing-rate model. The architecture of our model is shown in Figure 1. The STN neurons

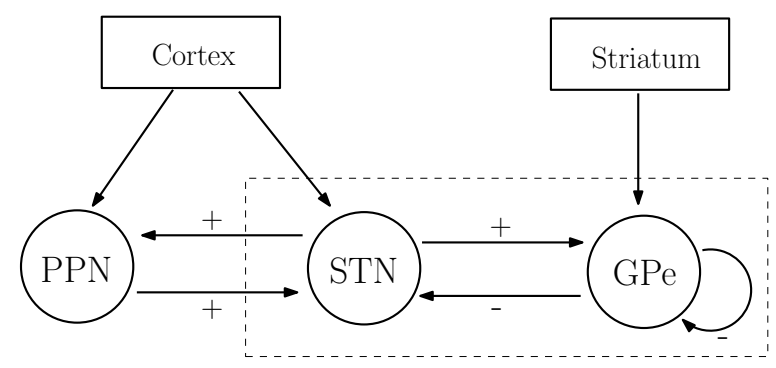

Figure 1: Schematic diagram of three neuclei: STN, GPe and PPN.

project excitatory axons to the GPe [8], while GPe neurons project inhibitory axons to the STN and to other GPe neurons [12]. The STN neurons project also excitatory axons to the PPN [7] which projects back excitatory axons to the STN [21]. Additionally, the STN and PPN nuclei receive inputs from cortex $[4,12,21]$ and the GPe nucleus receives input from the striatum [12]. The firing rates model of the STN, GPe and PPN populations respectively, are ruled by the delayed differential equations

$$
\begin{aligned}
& \tau_{s} \dot{x}_{s}=S_{s}\left(c_{s}^{p} x_{p}\left(t-\delta_{s}^{p}\right)-c_{s}^{g} x_{g}\left(t-\delta_{s}^{g}\right)+u_{s}\right)-x_{s} \\
& \tau_{g} \dot{x}_{g}=S_{g}\left(c_{g}^{s} x_{s}\left(t-\delta_{g}^{s}\right)-c_{g}^{g} x_{g}\left(t-\delta_{g}^{g}\right)+u_{g}\right)-x_{g} \\
& \tau_{p} \dot{x}_{p}=S_{p}\left(c_{p}^{s} x_{s}\left(t-\delta_{p}^{s}\right)+u_{p}\right)-x_{p}
\end{aligned}
$$

where $x_{s}, x_{g}$ and $x_{p}{ }^{1}$ represent the firing rates of the STN, GPe and PPN, respectively. The positive gains $c_{s}^{p}, c_{p}^{s}, c_{s}^{g}, c_{g}^{s}$ and $c_{g}^{g}$ define the weight of the different synaptic interconnections between these three neuron populations. The variables $u_{s}, u_{g}$ and $u_{p}$ describe the external inputs, from the striatum and cortex, received by these populations. The time constants $\tau_{s}, \tau_{g}$ and $\tau_{p}$ describe how rapidly the three populations react to the inputs. The scalar functions $S_{s}, S_{g}$ and $S_{p}$ define the activation functions of STN, GPe and PPN respectively. We assume that all the delays $\delta_{s}^{p}, \delta_{p}^{s}, \delta_{s}^{g}, \delta_{g}^{s}$ and $\delta_{g}^{g}$ are nonnegative and constant. We also assume the following on the activation functions.

Assumption 1 For each $i \in\{s, g, p\}$, the activation function $S_{i}: \mathbb{R} \rightarrow(0 ; 1)$ is continuously differentiable and strictly increasing. Its infimum is equal to 0 and its supremum is equal to 1 . In addition, its derivative $S_{i}^{\prime}$ is upper-bounded and there exists at least one point at which it reaches its maximum denoted $\sigma_{i}$.

A similar firing-rate model was exploited in [10, 18], to study the generation of pathological oscillations within the STN-GPe network. The remaining is devoted to the influence of PPN in this oscillations generation.

\footnotetext{
${ }^{1}$ By abuse of notation, we omit the dependency of $\dot{x}_{i}$ and $x_{i}$ on $t$, for $i \in\{s, g, p\}$.
} 


\section{Analysis in the absence of delays}

\subsection{Existence and uniqueness of equilibrium points}

The system (1) is defined everywhere on $\mathbb{R}^{3}$, since the activation functions are defined on $\mathbb{R}$. Nevertheless, one can check that the unit cube is invariant for this system.

Lemma 1 Under Assumption 1, for any constant inputs $u_{s}, u_{g}$ and $u_{p}$, the unit cube

$$
D:=\left\{\left(x_{s}, x_{g}, x_{p}\right) \in \mathbb{R}^{3}: x_{s}, x_{g}, x_{p} \in[0,1]\right\}
$$

is positively invariant for the delayed system (1).

The following result analyzes the existence and multiplicity of equilibria of the dynamics (1).

Theorem 1 Under Assumption 1, if

$$
\sigma_{p} \sigma_{s} c_{s}^{p} c_{p}^{s} \leq 1
$$

then the system (1) has a unique equilibrium point, for each constant vector $\left(u_{s}^{\star}, u_{g}^{\star}, u_{p}^{\star}\right) \in \mathbb{R}^{3}$. Otherwise, there exists a constant vector $\left(u_{s}^{\star}, u_{g}^{\star}, u_{p}^{\star}\right)$ for which the system (1) has at least three distinct equilibria. Moreover, if

$$
\left(\sigma_{p} c_{s}^{p} c_{p}^{s}-\frac{1}{\sigma_{s}}\right)\left(c_{g}^{g}+\frac{1}{\sigma_{g}}\right)>c_{s}^{g} c_{g}^{s}
$$

then, for each constant input $u_{g}^{\star}$, there exists a pair of constant inputs $\left(u_{s}^{\star}, u_{p}^{\star}\right)$ for which the system (1) has at least three distinct equilibria.

Theorem 1 generalizes the equilibrium study given by [18, Theorem 1] for a two-dimensional system describing the dynamics of two interacting subpopulations of excitatory and inhibitory neurons. It shows, in particular, that under condition (3), the existence of multiple equilibria can occur even for arbitrarily small striatal input.

\subsection{Stability of equilibria}

Consider an equilibrium point $x^{\star}$, associated to a vector of inputs $u^{\star}$, whose existence is ensured by Theorem 1. Let $e:=x-x^{\star}$ and $v:=u-u^{\star}$. The linearization of the dynamics (1) around $x^{\star}$ is given by:

$$
\begin{aligned}
& \tau_{s} \dot{e}_{s}=\sigma_{s}^{\star}\left(c_{s}^{p} e_{p}\left(t-\delta_{s}^{p}\right)-c_{s}^{g} e_{g}\left(t-\delta_{s}^{g}\right)+v_{s}\right)-e_{s} \\
& \tau_{g} \dot{e}_{g}=\sigma_{g}^{\star}\left(c_{g}^{s} e_{s}\left(t-\delta_{g}^{s}\right)-c_{g}^{g} e_{g}\left(t-\delta_{g}^{g}\right)+v_{g}\right)-e_{g} \\
& \tau_{p} \dot{e}_{p}=\sigma_{p}^{\star}\left(c_{p}^{s} e_{s}\left(t-\delta_{p}^{s}\right)+v_{p}\right)-e_{p},
\end{aligned}
$$

where

$$
\begin{aligned}
\sigma_{s}^{\star} & :=S_{s}^{\prime}\left(c_{s}^{p} x_{p}^{\star}-c_{s}^{g} x_{g}^{\star}+u_{s}^{\star}\right) \\
\sigma_{g}^{\star} & :=S_{g}^{\prime}\left(c_{g}^{s} x_{s}^{\star}-c_{g}^{g} x_{g}^{\star}+u_{g}^{\star}\right) \\
\sigma_{p}^{\star} & :=S_{p}^{\prime}\left(c_{p}^{s} x_{s}^{\star}+u_{p}^{\star}\right) .
\end{aligned}
$$

We next rely on this linearization to study the stability properties of $x^{\star}$. We start by considering the system (1) in the absence of delays.

Proposition 1 Consider the system (1) where all delays are zero, namely

$$
\delta_{i}^{j}=0 \quad \forall i, j \in\{s, g, p\} .
$$

Fix any input vector $u^{\star}:=\left(u_{s}^{\star}, u_{g}^{\star}, u_{p}^{\star}\right)^{T} \in \mathbb{R}^{3}$, consider an equilibrium $x^{\star}:=\left(x_{s}^{\star}, x_{g}^{\star}, x_{p}^{\star}\right)^{T}$ associated to these inputs and let $\sigma_{i}^{\star}, i \in\{s, g, p\}$, be defined by (5). Then, under Assumption 1, the following holds: 
A)

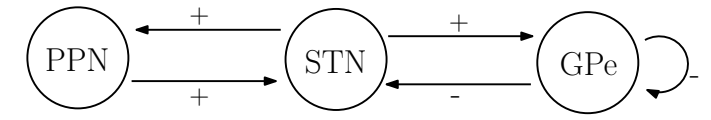

B)

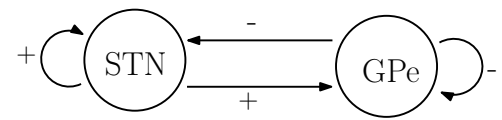

Figure 2: A) three neuron populations. B) two neuron populations.

- If the conditions

$$
\begin{gathered}
\left(\sigma_{p}^{\star} c_{s}^{p} c_{p}^{s}-\frac{1}{\sigma_{s}^{\star}}\right)\left(c_{g}^{g}+\frac{1}{\sigma_{g}^{\star}}\right)<c_{s}^{g} c_{g}^{s} \\
\frac{\sigma_{s}^{\star}}{\tau_{s}+\tau_{p}}\left(\sigma_{p}^{\star} c_{s}^{p} c_{p}^{s}-\frac{1}{\sigma_{s}^{\star}}\right)<\frac{\sigma_{g}^{\star}}{\tau_{g}}\left(c_{g}^{g}+\frac{1}{\sigma_{g}^{\star}}\right)
\end{gathered}
$$

are both satisfied, then the equilibrium point $x^{\star}$ is locally exponentially stable for (1).

- If the conditions

$$
\begin{array}{r}
\sigma_{p} \sigma_{s} c_{s}^{p} c_{p}^{s} \leq 1 \\
\sigma_{s}\left(c_{s}^{p}+c_{s}^{g}\right)+\sigma_{g} c_{g}^{s}+\sigma_{p} c_{p}^{s}<2
\end{array}
$$

are both satisfied then $x^{\star}$ is globally asymptotically stable for (1).

Section 4 is devoted to the study of the preservation of these stability properties in the presence of delays. Before that, we close this section by underlining some similarities with the two-nuclei approach developed in $[10,19,18]$.

\subsection{Comparison with an excitatory-inhibitory model}

The PPN-STN interaction being excitatory in both directions, a natural question is whether the threedimensional system (1) can be analyzed by a two-dimensional system in which the STN feedback to itself in an excitatory manner. Both these situations are depicted by Figure 2. This question creates a natural bridge between this work and $[10,18,19]$ in which no explicit influence of PPN is considered. In the situation described by Figure 2B, the evolution of GPe and STN are given by

$$
\begin{aligned}
& \tau_{s} \dot{x}_{s}=S_{s}\left(c_{s}^{s} x_{s}\left(t-\delta_{s}^{s}\right)-c_{s}^{g} x_{g}\left(t-\delta_{s}^{g}\right)+u_{s}\right)-x_{s} \\
& \tau_{g} \dot{x}_{g}=S_{g}\left(c_{g}^{s} x_{s}\left(t-\delta_{g}^{s}\right)-c_{g}^{g} x_{g}\left(t-\delta_{g}^{g}\right)+u_{g}\right)-x_{g}
\end{aligned}
$$

where $c_{s}^{s}$ defines the STN self-feedback and $\delta_{s}^{s}$ denotes its transmission delay.

The following result provides conditions under which the networks of Figure $2 \mathrm{~A}$ and Figure $2 \mathrm{~B}$ have the same number of equilibria.

Proposition 2 Under Assumption 1, the two following facts hold:

- Under the condition that $c_{s}^{s} \leq \sigma_{p} c_{s}^{p} c_{p}^{s}$, if for every input $\left(u_{s}^{\star}, u_{g}^{\star}, u_{p}^{\star}\right)$ system (1) has unique equilibrium then for every input $\left(u_{s}^{\star}, u_{g}^{\star}\right)$ system (11) has unique equilibrium.

- Under the condition that $c_{s}^{s} \geq \sigma_{p} c_{s}^{p} c_{p}^{s}$, if for every input $\left(u_{s}^{\star}, u_{g}^{\star}\right)$ system (11) has unique equilibrium then for every input $\left(u_{s}^{\star}, u_{g}^{\star}, u_{p}^{\star}\right)$ system (1) has unique equilibrium. 
The following result provides additional conditions under which global asymptotic stability is preserved between systems (1) and (11).

Proposition 3 Suppose that $\sigma_{s} c_{s}^{s} \leq 1$ and that conditions (9)-(10) are verified. Under Assumption 1, if

$$
\sigma_{s} c_{s}^{s} \leq \frac{1}{2}\left(\sigma_{s} c_{s}^{p}+\sigma_{p} c_{p}^{s}\right)
$$

then the global asymptotic stability of (1) implies the global asymptotic stability of (11).

\section{Robustness to delays}

In this section, we study the stability properties of the equilibria of (1) by relying on its linearization (4) around an equilibrium point. This system can be described in the frequency domain using the closed-loop transfer functions

$$
\begin{aligned}
& H_{s}(s)=\frac{\sigma_{s}^{\star}}{\tau_{s} s+1}, \quad H_{p}(s)=\frac{\sigma_{p}^{\star}}{\tau_{p} s+1} \\
& H_{g}(s)=\frac{\sigma_{g}^{\star}}{\tau_{g} s+1+\sigma_{g}^{\star} c_{g}^{g} e^{-\delta_{g}^{g} s}},
\end{aligned}
$$

by the following system

$$
\begin{aligned}
& \frac{1}{H_{s}} E_{s}+c_{s}^{g} e^{-\delta_{s}^{g} s} E_{g}-c_{s}^{p} e^{-\delta_{s}^{p} s} E_{p}=V_{s} \\
& \frac{1}{H_{g}} E_{g}-c_{g}^{s} e^{-\delta_{g}^{s} s} E_{s}=V_{g} \\
& \frac{1}{H_{p}} E_{p}-c_{p}^{s} e^{-\delta_{p}^{s} s} E_{s}=V_{p}
\end{aligned}
$$

where $E:=\left(E_{s}, E_{g}, E_{p}\right)^{T}$, and $V:=\left(V_{s}, V_{g}, V_{p}\right)^{T}$ are the Laplace transforms of $e$ and $v$, respectively. The system defined by (14) can be written as the following feedback system $(G, K)$

$$
\left\{\begin{array}{l}
E=G E^{\prime}+U \\
E^{\prime}=K E \\
U=G V
\end{array}\right.
$$

where the two transfer matrices $G$ and $K$ are given by the following

$$
\begin{gathered}
G(s):=\left(\begin{array}{ccc}
H_{s}(s) & 0 & 0 \\
0 & H_{g}(s) & 0 \\
0 & 0 & H_{p}(s)
\end{array}\right), \\
K(s):=\left(\begin{array}{ccc}
0 & -c_{s}^{g} e^{-\delta_{s}^{g} s} & c_{s}^{p} e^{-\delta_{s}^{p} s} \\
c_{g}^{s} e^{-\delta_{g}^{s} s} & 0 & 0 \\
c_{p}^{s} e^{-\delta_{p}^{s} s} & 0 & 0
\end{array}\right) .
\end{gathered}
$$

In order to study the stability properties of the feedback system (15), we make use of the Nyquist Theorem [5, Theorem 9.1.8] for MIMO delayed systems. We stress that here the Nyquist Theorem is applied in its general form for the Callier-Desoer class of scalar irrational transfer functions $\hat{\mathcal{B}}$ (see Section A.1 for definitions, see also [5, Definitions 7.1.4 and 7.1.6] for more details). We start by checking the stability of the transfer matrices $G$ and $K$. Noticing that $G$ and $K$ are irrational transfer matrices, we begin by verifying that each of its components belongs to $\hat{\mathcal{B}}$. This is stated by the following result. 


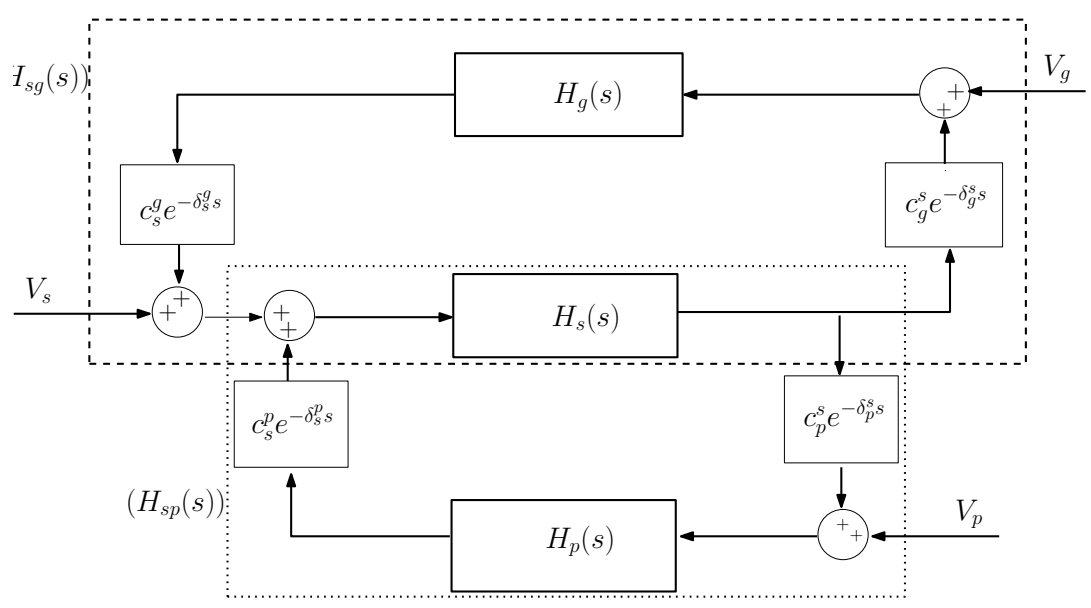

Figure 3: Bloc diagram of the linearized system (4).

Proposition 4 The entries of the transfer matrices $G$ and $K$ defined in (16)-(17) all belong to the Callier-Desoer class of scalar irrational transfer functions $\hat{\mathcal{B}}$.

The transfer matrix $G$ is not necessarily stable. This comes from the fact that the transfer function $H_{g}$ is not necessarily stable. However, one can observe that $H_{g}$ is always stable when $\delta_{g}^{g}=0$. In [18, Lemma 3], the authors establishes the existence of a delay margin $\Delta\left(H_{g}\right)$ of $H_{g}$ such that the transfer function $H_{g}$ is input-output stable if and only if $\delta_{g}^{g}<\Delta\left(H_{g}\right)$. We recall that the delay margin of a SISO transmittance $^{2} H \in \hat{\mathcal{B}}$, is defined by:

$$
\Delta(H):=\sup \left\{\bar{\tau}>0: \text { the feedback }\left(H, e^{-\tau s}\right) \text { is input-output stable } \forall \tau \in[0, \bar{\tau})\right\} .
$$

To compute the delay margin of $H$ one can associate, at a given frequency $\omega$, its gain $\gamma_{H}(\omega)$ and phase $\varphi_{H}(\omega)$ which are defined by the relations

$$
\gamma_{H}(\omega):=20 \log _{10}|H(i \omega)| \text { and } \varphi_{H}(\omega):=\arg (H(i \omega)) .
$$

In our approach, the case where the function $\gamma_{H}$ is strictly decreasing is of a particular interest. Indeed, in this case, to any strictly proper transfer function $H$ such that $\gamma_{H}(0)>0$ we can associate its (gain) crossover frequency $\omega_{H}$, which is defined as the only frequency such that

$$
\gamma_{H}\left(\omega_{H}\right)=0 .
$$

This frequency can be used to define the delay margin $\Delta(H)$ by the relationship

$$
\Delta(H)=\frac{\pi-\varphi_{H}\left(\omega_{H}\right)}{\omega_{H}} .
$$

If $\gamma_{H}$ is strictly decreasing but $\gamma_{H}(0) \leq 0$, and if $H$ is minimum phase (it has neither unstable poles nor unstable zeroes), then we can still define $\Delta(H)=+\infty$.

Since the stability properties of $G$ and $K$ are fully known in open loop, we now turn to study the stability of the feedback system (15). The following result provides a necessary and sufficient condition for the stability of the feedback system (15). Its statement relies on the following two transfer functions

where the quantities

$$
K_{p}(s):=c_{p} H_{p}(s) e^{-\delta_{p} s} \quad \text { and } \quad K_{g}(s):=-c_{g} H_{g}(s) e^{-\delta_{g} s}
$$

$$
\begin{array}{lll}
c_{p}:=c_{s}^{p} c_{p}^{s}, & c_{g}:=c_{s}^{g} c_{g}^{s}, \\
\delta_{p}:=\delta_{p}^{s}+\delta_{s}^{p}, & \delta_{g}:=\delta_{g}^{s}+\delta_{s}^{g}
\end{array}
$$

are defined in order to obtain a lighter notation.

\footnotetext{
${ }^{2}$ For a formal definition of input-output stability, see Definition 1 in Section A.2, see also [5, Definition 9.1.1].
} 
Proposition 5 Suppose that $H_{g}$ is input-output stable. The feedback system defined by (15) is inputoutput stable if and only if

$$
\operatorname{ind}\left(1-H_{s}\left(K_{p}+K_{g}\right)\right)=0,
$$

where ind $\left(1-H_{s}\left(K_{p}+K_{g}\right)\right)$ denotes the Nyquist index ${ }^{3}$ [5, Definition A.1.15, p. 569] of $1-H_{s}\left(K_{p}+K_{g}\right)$.

The proof of this result is based on standard arguments of the closed-loop stability of delayed MIMO system [5].

The bloc diagram (see Figure 3) comprises two closed-loops, one with external input $\left(V_{s}, V_{g}\right)$ and the other with external input $\left(V_{s}, V_{p}\right)$. Let us introduce the two following transfer functions

$$
H_{s p}:=\frac{H_{s}}{1-H_{s} K_{p}} \quad \text { and } \quad H_{s g}:=\frac{H_{s}}{1-H_{s} K_{g}} .
$$

$H_{s p}$ and $H_{s g}$ are the closed-loop transfer functions of the rectangles marked in dotted and dashed respectively and which are calculated between $V_{s}$ and $E_{s}$.

The following lemma states that the stability analysis of the full network can be reduced to the study of either $H_{s g} K_{p}$ or $H_{s p} K_{g}$.

Lemma 2 Suppose that $H_{g}$ is input-output stable. If the feedback systems $\left(H_{s}, K_{p}\right)$ and $\left(H_{s}, K_{g}\right)$ are input-output stable, then we have

$$
\operatorname{ind}\left(1-H_{s}\left(K_{p}+K_{g}\right)\right)=\operatorname{ind}\left(1-H_{s g} K_{p}\right)=\operatorname{ind}\left(1-H_{s p} K_{g}\right) .
$$

According to Lemma 2, if the transfer functions $H_{g}, H_{s p}$ and $H_{s g}$ are input-output stable, then the stability analysis of the feedback system (15) can be equivalently achieved by studying one of the two feedback systems $\left(H_{s p}, K_{g}\right)$ or $\left(H_{s g}, K_{p}\right)$. In what follows, we choose to focus on the feedback system $\left(H_{s p}, K_{g}\right)$ to study the stability of the network of Figure 3. This choice is motivated by the following lemma, which provides conditions under which the gain $\gamma_{H_{s p}}$ is monotonically decreasing, thus considerably simplifying the Nyquist plot analysis.

Lemma 3 Consider the transfer function $H_{s p}$ defined by (24). For each positive $\delta_{p}$ as defined in (22), there exists a positive gain $c_{p}^{\star}\left(\delta_{p}\right)$ such that the loop gain $\gamma_{H_{s p}}$ is strictly decreasing on $\mathbb{R}^{+}$for every $c_{p}=c_{s}^{p} c_{p}^{s} \in\left(0, c_{p}^{\star}\left(\delta_{p}\right)\right)$.

(A)

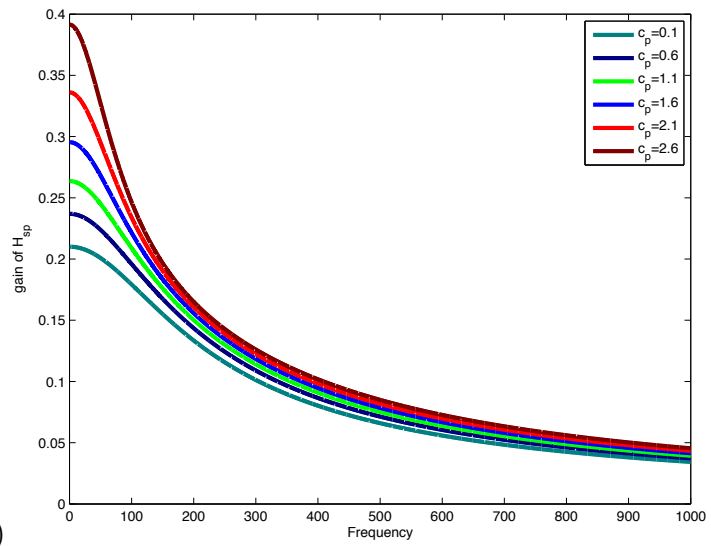

(B)

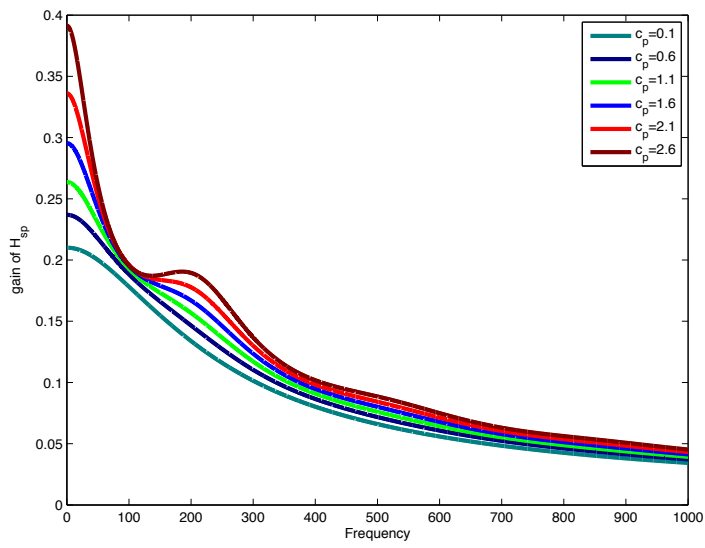

Figure 4: Influence of the connection weight $c_{p}$ on the monotonicity of the gain of $H_{s p}$ for $K=0.1$.

Figure 4 illustrates two different cases of Lemma 3. Figure $4 \mathrm{~A}$ is plotted for $\delta_{p}=2 \mathrm{~ms}$. In this case the value of $c_{p}^{\star}\left(\delta_{p}\right)$ is close to 1.8 . While Figure $4 \mathrm{~B}$ is plotted for $\delta_{p}=18 \mathrm{~ms}$, and in this case the value of

\footnotetext{
${ }^{3}$ Roughly speaking, 'ind' denotes the number of encirclements of the Nyquist plot of the transfer function around the origin in a counterclockwise sens as $s$ decreases from $i \infty$ to $-i \infty$ over the indented imaginary axis.
} 
$c_{p}^{\star}\left(\delta_{p}\right)$ is close to 0.55 .

In order to prove the stability of $H_{s p}$, we can directly invoke [18, Theorem 2]. While the stability of $H_{s p}$ does not depend on the loop delay $\delta_{p}$, this is not the case for $H_{s g}$. In particular, when $c_{g} \sigma_{g}^{\star} \sigma_{s}^{\star}>$ $1+c_{g}^{g} \sigma_{g}^{\star}$, only a finite upper bound on the delay $\delta_{g}$ can be tolerated. These two observations are formalized by the following statement.

Lemma 4 Consider the transfer functions $H_{s p}$ and $H_{s g}$, defined in (24), and let

$$
G_{s p}:=c_{p} H_{s} H_{p} \quad \text { and } \quad G_{s g}:=-c_{g} H_{s} H_{g},
$$

where $H_{s}, H_{g}$ and $H_{p}$ are defined in (13). Consider the constants $\sigma_{i}^{\star}, i \in\{s, p, g\}$, defined by (5). Then following facts hold:

- The transfer function $H_{s p}$ is input-output stable if and only if $\delta_{p}<\Delta\left(G_{s p}\right)$. If $c_{p} \sigma_{p}^{\star} \sigma_{s}^{\star}<1$ then $\Delta\left(G_{s p}\right)=+\infty$, otherwise $\Delta\left(G_{s p}\right) \leq 0$.

- Assuming that the gain $\gamma_{H_{s g}}$ is strictly decreasing, the transfer function $H_{s g}$ is input-output stable if and only if $\delta_{g}<\Delta\left(G_{s g}\right)$. If the inequality $c_{g} \sigma_{g}^{\star} \sigma_{s}^{\star}<1+c_{g}^{g} \sigma_{g}^{\star}$ then $\Delta\left(G_{s g}\right)=+\infty$, otherwise $\Delta\left(G_{s g}\right) \in(0 ;+\infty)$.

(A)
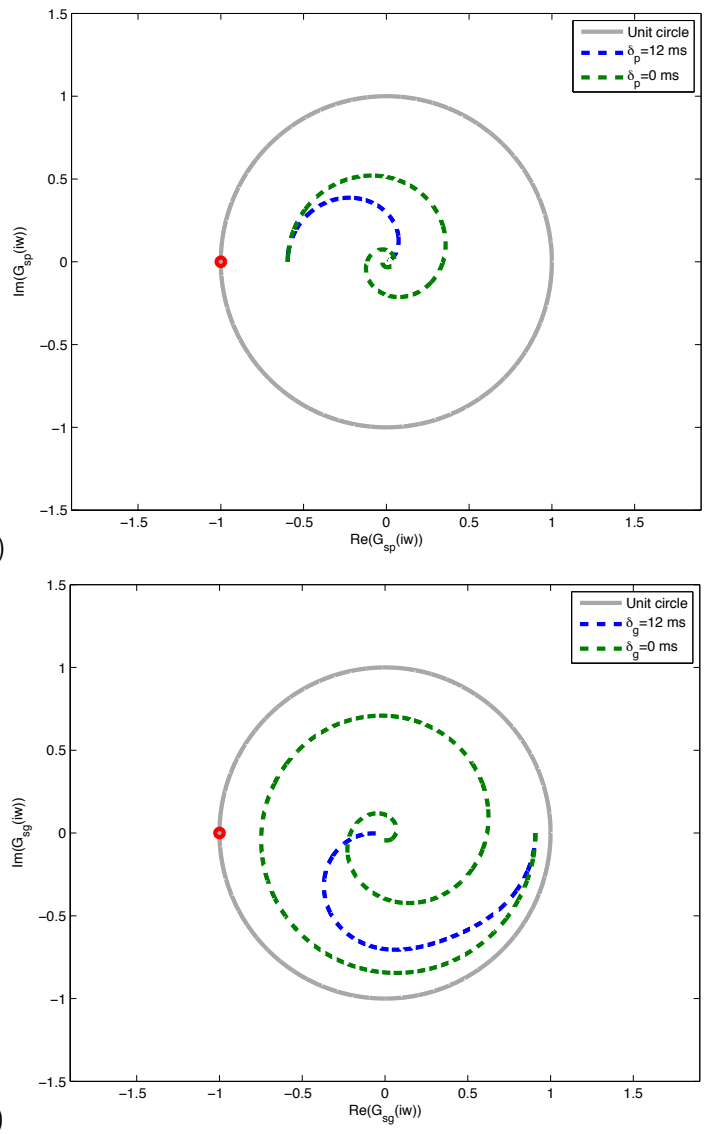

(B)

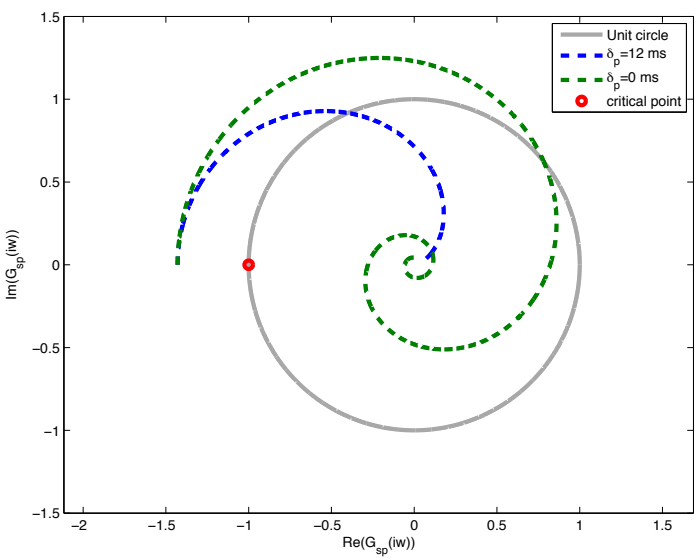

(D)

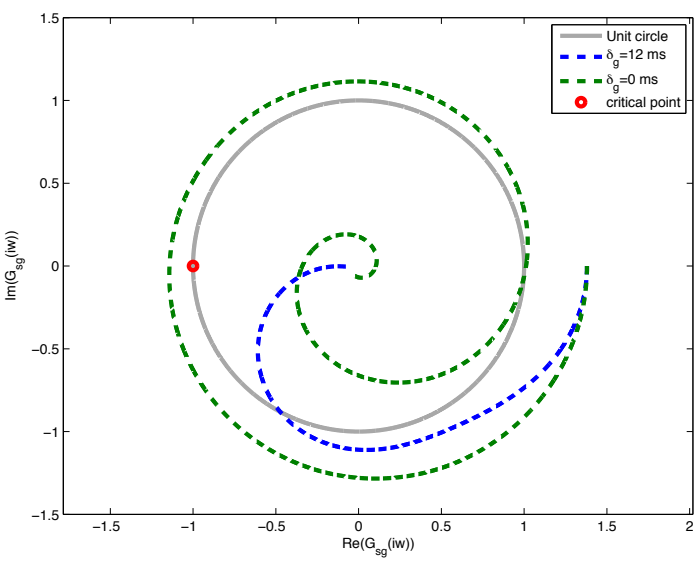

Figure 5: Nyqyuist diagram associated to four different cases of Lemma 4.

Figures 5A and 5C illustrate the two cases of Lemma 4, where the stability of $H_{s p}$ and $H_{s g}$ does not depend on the value of $\delta_{p}$ and $\delta_{g}$, respectively. While, Figures 5B and 5D illustrate the two others cases, where the stability of $H_{s p}$ is lost and that of $H_{s g}$ is positive, respectively. 
With Proposition 5 and Lemmas 2 and 4 at hand, we are now ready to state the following result, which provides conditions for the local asymptotic stability of the linear system (4).

Theorem 2 Consider the delayed differential equation defined by (4). Let $u^{\star} \in \mathbb{R}^{3}$ be any input such that, for the equilibrium $x^{\star}$ associated to these inputs, the transfer functions $H_{g}$ and $H_{s p}$, defined in (13)(24), are input-output stable. Define $H:=c_{g} H_{g} H_{s p}$. Assume that the gain of $\gamma_{H}$ is strictly decreasing (which can be verified using Lemma 3 and [18, Lemma 4] together). For every $\delta_{p}>0, x^{\star}$ is exponentially stable for (4) if and only if $\delta_{g}<\Delta(H)$.

Using Lemma 3 and the fact that the transfer function $H_{g}$ can be strictly decreasing [18, Lemma 4], the following result derives directly from Theorem 2 .

Corollary 1 Consider the delayed differential equation defined by (4). Let $u^{\star} \in \mathbb{R}^{3}$ be any input such that, for the equilibrium $x^{\star}$ associated to these inputs, the transfer functions $H_{g}$ and $H_{s p}$ are input-output stable. Assume that the gain of $\gamma_{H_{g}}$ is strictly decreasing. Then, for each $\delta_{p}>0$, there exist $c_{p}^{\star}>0$ such that for each $c_{p}<c_{p}^{\star}, x^{\star}$ is exponentially stable for (4) if and only if $\delta_{g}<\Delta(H)$.

\section{$5 \quad$ Numerical simulations}

We now validate our theoretical findings trough numerical simulations. As in [18], the activation functions $S_{s}, S_{g}$ and $S_{p}$ are approximated by a normalized sigmoïd function of the form

$$
S_{i}(x)=\frac{B_{i}}{B_{i}+\left(M_{i}-B_{i}\right) e^{-4 x}}, \quad \forall i \in\{s, g, p\}
$$

where $B_{i}$ and $M_{i}$ are given in Table 1 . These functions satisfy Assumption 1 with $\sigma_{i}=1$, for $i \in\{s, g, p\}$. The parameter values of system (1), and precisely the transmission delays, time-constants, and activation functions, are chosen as follows. For the STN and GPe nuclei, these values are the same as those taken in $[10,18]$ and they are given in Table 1 . For the PPN, and since we did not find exact parameters in the literature, the parameters are taken equal to those that correspond to the STN. As in [10, 18] the interconnection gain $c_{j}^{i}$ from nucleus $i$ to nucleus $j(i, j \in\{s, g\})$ in the STN-GPe network, is given by:

$$
c_{j}^{i}=c_{j}^{i H}+k\left(c_{j}^{i^{D}}-c_{j}^{i H}\right) \quad \forall i, j \in\{s, g\}
$$

where $k$ is a parameter that describes the evolution of Parkinson's disease, and ${c_{j}^{i}}^{H}$ and ${c_{j}^{i}}^{D}$ are, respectively, the interconnection gains for the healthy and diseased states (given in Table 2). Similarly, the external inputs are given by:

$$
u_{i}=u_{i}{ }^{H}+k\left(u_{i}{ }^{D}-u_{i}{ }^{H}\right) \quad \forall i \in\{s, g, p\}
$$

where $u_{i}{ }^{H}$ and $u_{i}{ }^{D}$ are, respectively, the external inputs for the healthy and diseased states (given in Table 2). The external inputs to the PPN, for the healthy and diseased state, are taken equal to those of STN. The parameter $k$ is fixed to $k=0.2$, value for which the two-dimensional system STN-GPe is locally asymptotically stable $[10,18,19]$. The evolution of system (1) is carried out in function of the interconnection gains $c_{p}^{s}$ and $c_{s}^{p}$, which are taken equal.

One can easily check the dependency on the parameter $c_{p}$ (defined in $(22)$ ) of the delay margin $\Delta(H)$, by plotting $\Delta(H)$ as a function of $c_{p} \in(0 ; 1)$ (see Figure 6 ). It can be observed that the delay margin decreases when $c_{p}$ increases. In addition, one can see that when $c_{p}=0.2$ the linear system (4) is approximately at the bifurcation point. After checking the dependency on $c_{p}$ of $\Delta(H)$, we set two distinct values of $c_{p}$ around the bifurcation point: $c_{p}=0.1$ and $c_{p}=0.3$ (note that for these values the gain $\gamma_{H_{s p}}$ is strictly decreasing). We simulate the evolution of nonlinear system (1) together with Nyquist diagram of its linearization (4) in both cases. The results of our simulations are presented in Figure 7A and 7B. When $c_{p}=0.1$, we have $\Delta(H)>\delta_{g}$, the Nyquist plot does not encircles the critical point, and the nonlinear system (1) is stable. When $c_{p}=0.3$, we have $\Delta(H)<\delta_{g}$, the critical point is encircled, and the nonlinear system (1) is unstable, which predicts the birth of pathological oscillations within the PPN-STN-GPe network. 
Table 1: Parameter values needed for simulation.

\begin{tabular}{ccc}
\hline Parameter & Value & Description \\
\hline$\delta_{g}^{s}$ & $6 \mathrm{~ms}$ & Delay from STN to GPe \\
$\delta_{s}^{g}$ & $6 \mathrm{~ms}$ & Delay from GPe to STN \\
$\delta_{p}^{s}$ & $6 \mathrm{~ms}$ & Delay from STN to PPN \\
$\delta_{s}^{p}$ & $6 \mathrm{~ms}$ & Delay from PPN to STN \\
$\delta_{g}^{g}$ & $4 \mathrm{~ms}$ & Internal self-inhibition delay in the GPe \\
$\tau_{s}$ & $6 \mathrm{~ms}$ & STN time constant \\
$\tau_{g}$ & $14 \mathrm{~ms}$ & GPe time constant \\
$\tau_{p}$ & $6 \mathrm{~ms}$ & PPN time constant \\
$M_{s}$ & $300 \mathrm{spk} / \mathrm{s}$ & STN Maximal firing rate \\
$B_{s}$ & $17 \mathrm{spk} / \mathrm{s}$ & Firing rate at rest for STN \\
$M_{g}$ & $400 \mathrm{spk} / \mathrm{s}$ & GPe Maximal firing rate \\
$B_{g}$ & $75 \mathrm{spk} / \mathrm{s}$ & Firing rate at rest for GPe \\
$M_{p}$ & $300 \mathrm{spk} / \mathrm{s}$ & PPN Maximal firing rate \\
$B_{p}$ & $17 \mathrm{spk} / \mathrm{s}$ & Firing rate at rest for PPN \\
\hline
\end{tabular}

Table 2: Parameter values needed for simulation.

\begin{tabular}{ccc}
\hline Parameter & Healthy state & Diseased state \\
\hline$c_{g}^{s}$ & 14.3 & 15 \\
$c_{s}^{g}$ & 1.5 & 14.3 \\
$c_{g}^{g}$ & 6.6 & 12.3 \\
$u_{s}$ & 0.2 & 0.8 \\
$u_{g}$ & 0.1 & 0.7 \\
$u_{p}$ & 0.2 & 0.8 \\
\hline
\end{tabular}

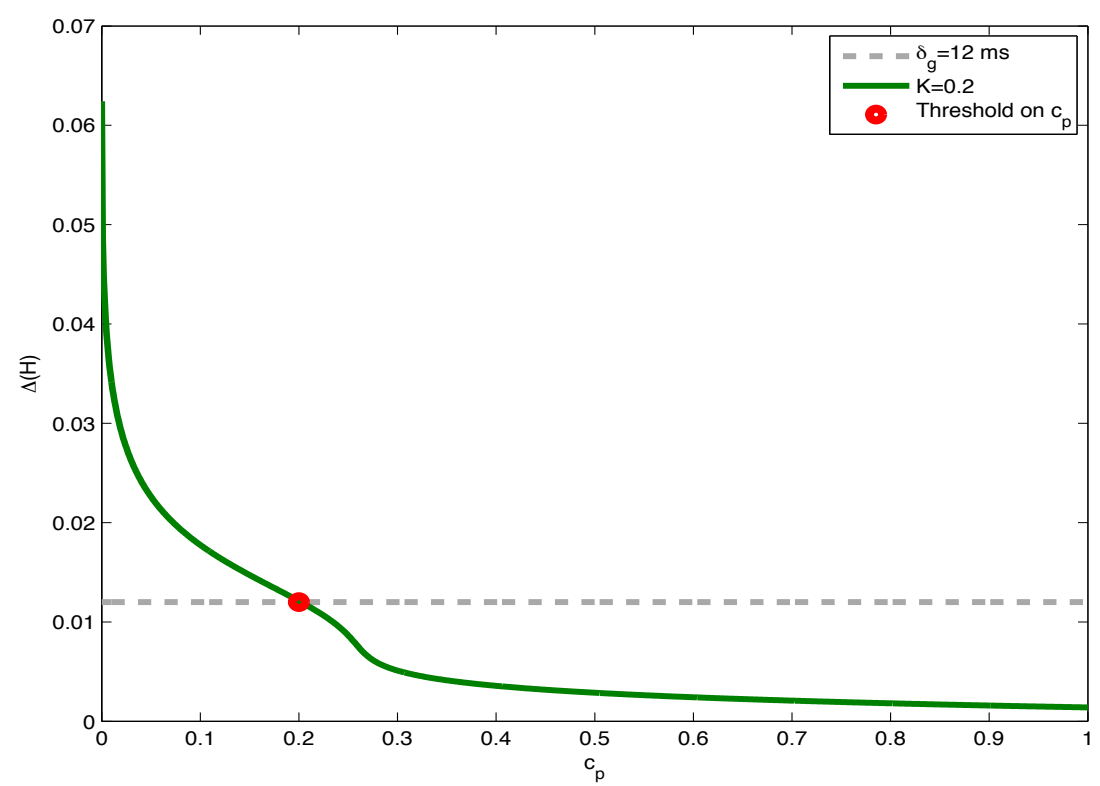

Figure 6: Influence of $c_{p}$ on the delay margin $\Delta(H)$, for $k=0.2$. 

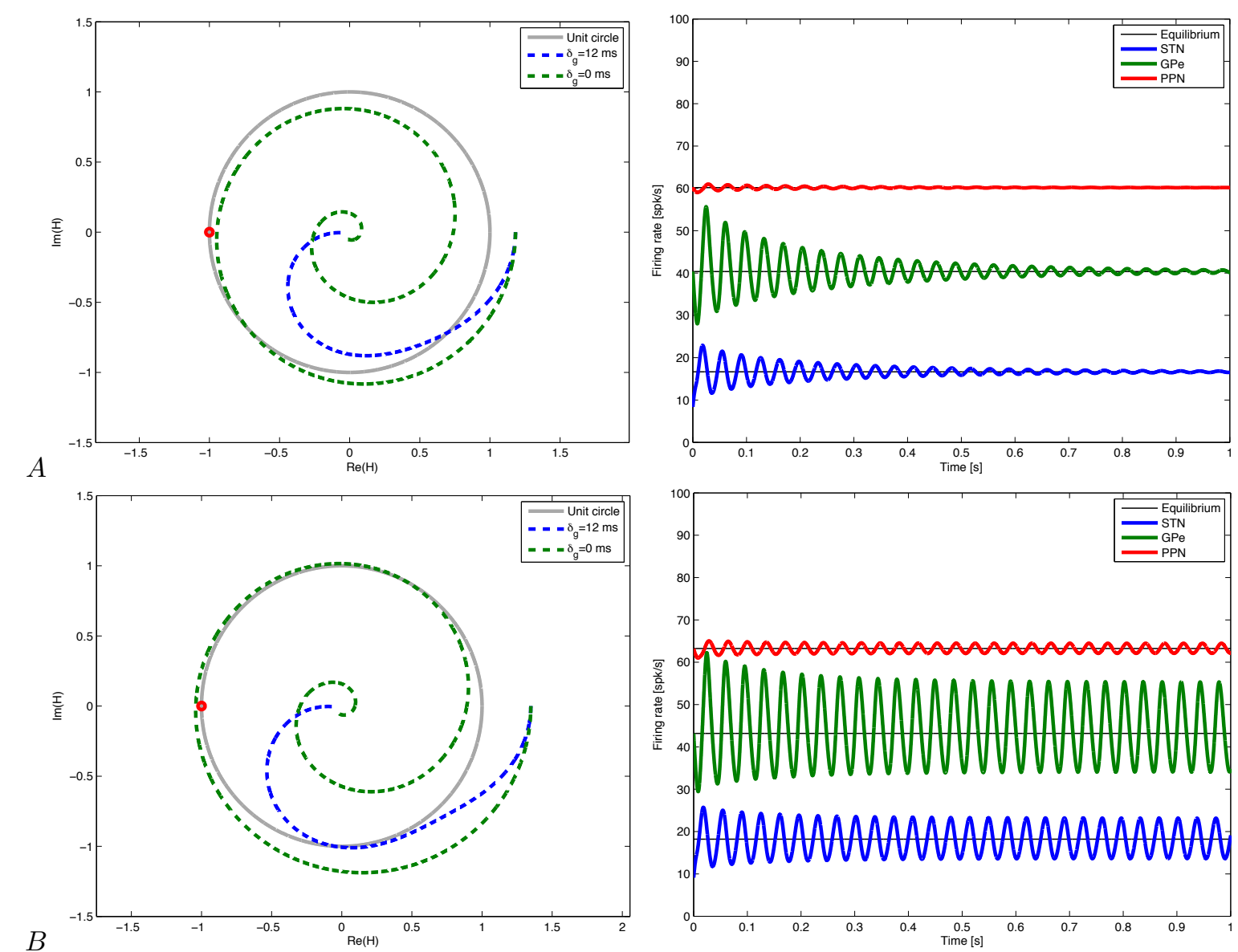

Figure 7: Influence on stability of the interconnection gain $c_{p}^{s}$ and $c_{s}^{p}$, for $k=0.2$. On the left, the open-loop frequency-response is represented in a Nyquist diagram. On the right, the temporal evolution of the system (1) is plotted.

\section{Mathematical proofs}

\subsection{Proof of Theorem 1}

Proof of Lemma 1 By Assumption 1, for $i \in\{s, g, p\}$, the activation functions satisfy

$$
S_{i}(x) \geq 0 \text { and } S_{i}(x) \leq 1 \text { for each } x \in[0,1] .
$$

Therefore, if $x_{i} \leq 0$ then $\dot{x}_{i} \geq 0$ and if $x_{i} \geq 1$ then $\dot{x}_{i} \leq 0$. It follows that, for each point on the boundary of $D$, the vector field that defines the system dynamics points towards $D$. Hence, this set is positively invariant (independently of the values of the delays and of the external inputs).

Proof of Theorem 1 First step. For any constant inputs $u_{s}^{\star}, u_{g}^{\star}$ and $u_{p}^{\star}$ consider the three isoclines

$$
\begin{aligned}
& N_{s}:=\left\{\left(x_{s}, x_{g}, x_{p}\right) \in \mathbb{R}^{3}: x_{s}=S_{s}\left(c_{s}^{p} x_{p}-c_{s}^{g} x_{g}+u_{s}^{\star}\right)\right\} \\
& N_{g}:=\left\{\left(x_{s}, x_{g}, x_{p}\right) \in \mathbb{R}^{3}: x_{g}=S_{g}\left(c_{g}^{s} x_{s}-c_{g}^{g} x_{g}+u_{g}^{\star}\right)\right\} \\
& N_{p}:=\left\{\left(x_{s}, x_{g}, x_{p}\right) \in \mathbb{R}^{3}: x_{p}=S_{p}\left(c_{p}^{s} x_{s}+u_{p}^{\star}\right)\right\} .
\end{aligned}
$$


All equilibria $\left(x_{s}^{\star}, x_{g}^{\star}, x_{p}^{\star}\right)$ of system (1) are located on the set $N_{s} \cap N_{g} \cap N_{p}$. Observe that these sets do not depend on the system's delays. Since

$$
N_{i} \subset\left\{\left(x_{s}, x_{g}, x_{p}\right) \in \mathbb{R}^{3}: x_{i} \in[0,1]\right\} \text { for each } i \in\{s, g, p\},
$$

it follows that all equilibria belong to the unit cube.

Second step. Using the inverse activation functions $T_{i}$ defined above, the isoclines (28) can be described by:

$$
\begin{aligned}
& N_{s}=\left\{\left(x_{s}, x_{g}, x_{p}\right) \in \mathbb{R}^{3}: x_{g}=\frac{1}{c_{s}^{g}}\left(c_{s}^{p} x_{p}-T_{s}\left(x_{s}\right)+u_{s}^{\star}\right)\right\} \\
& N_{g}=\left\{\left(x_{s}, x_{g}, x_{p}\right) \in \mathbb{R}^{3}: x_{s}=\frac{1}{c_{g}^{s}}\left(c_{g}^{g} x_{g}+T_{g}\left(x_{g}\right)-u_{g}^{\star}\right)\right\} \\
& N_{p}=\left\{\left(x_{s}, x_{g}, x_{p}\right) \in \mathbb{R}^{3}: x_{p}=S_{p}\left(c_{p}^{s} x_{s}+u_{p}^{\star}\right)\right\} .
\end{aligned}
$$

The set $N_{g}$ can always be described as the graph of a strictly increasing function, defined on the interval $[0,1]$. That is

$$
x_{s}=\varphi\left(x_{g}, u_{g}^{\star}\right), \quad \forall x_{g} \in[0,1] .
$$

Where the function $\varphi:[0,1] \rightarrow \mathbb{R}$ is defined as

$$
\varphi\left(x_{g}, u_{g}^{\star}\right):=\frac{1}{c_{g}^{s}}\left(c_{g}^{g} x_{g}+T_{g}\left(x_{g}\right)-u_{g}^{\star}\right) .
$$

Using the fact that for each $u_{g}^{\star}$ the function $\varphi$ is both surjective and strictly increasing on the interval $[0,1]$, we can define $x_{g}^{0}\left(u_{g}^{\star}\right)$ and $x_{g}^{1}\left(u_{g}^{\star}\right)$ as the only solutions of the equations

$$
\varphi\left(x_{g}^{0}, u_{g}^{\star}\right)=0
$$

and

$$
\varphi\left(x_{g}^{1}, u_{g}^{\star}\right)=1
$$

respectively. Observe that a point $\left(x_{s}, x_{g}, x_{p}\right)$ is an equilibrium requires that $x_{g}$ belongs to the interval $\left[x_{g}^{0}, x_{g}^{1}\right]$ and satisfies the equation

$$
x_{g}=\frac{1}{c_{s}^{g}}\left[c_{s}^{p} S_{p}\left(c_{p}^{s} \varphi\left(x_{g}, u_{g}^{\star}\right)+u_{p}^{\star}\right)-T_{s}\left(\varphi\left(x_{g}, u_{g}^{\star}\right)\right)+u_{s}^{\star}\right] .
$$

Third step. We are now able to define, for each $x \in\left[x_{g}^{0}, x_{g}^{1}\right]$, the function

$$
L(x)=\frac{1}{c_{s}^{g}}\left[c_{s}^{p} S_{p}\left(c_{p}^{s} \varphi\left(x, u_{g}^{\star}\right)+u_{p}^{\star}\right)-T_{s}\left(\varphi\left(x, u_{g}^{\star}\right)\right)+u_{s}^{\star}\right]-x .
$$

By construction, if $\left(x_{s}, x_{g}, x_{p}\right)$ is an equilibrium point of system (1) then we have

$$
L\left(x_{g}\right)=0
$$

Moreover, noticing that $T_{s}(1)=+\infty$ and $T_{s}(0)=-\infty$, in any of the three cases described by Theorem 1 , the function $L$ is such that

$$
\lim _{x \rightarrow x_{g}^{0+}} L(x)=+\infty \quad \text { and } \quad \lim _{x \rightarrow x_{g}^{1-}} L(x)=-\infty .
$$

Since this function is continuous, the intermediate value theorem guarantees that it must vanish on $\left(x_{g}^{0}, x_{g}^{1}\right)$. If we omit the dependency of $\varphi(\cdot)$ and $\varphi^{\prime}(\cdot)$ in $u_{g}^{\star}$, the derivative of $L$ can be computed using the chain rule

$$
L^{\prime}(x)=\frac{1}{c_{s}^{g}}\left[c_{s}^{p} c_{p}^{s} \varphi^{\prime}(x) S_{p}^{\prime}\left(c_{p}^{s} \varphi(x)+u_{p}^{\star}\right)-T_{s}^{\prime}(\varphi(x)) \varphi^{\prime}(x)\right]-1
$$

with

$$
\varphi^{\prime}(x)=\frac{1}{c_{g}^{s}}\left(c_{g}^{g}+T_{g}^{\prime}(x)\right) .
$$


Grouping the factors of $\varphi^{\prime}(x)$ in $L^{\prime}(x)$ leads to

$$
L^{\prime}(x)=\frac{S_{p}^{\prime}\left(c_{p}^{s} \varphi(x)+u_{p}^{\star}\right)}{c_{g}^{s} c_{s}^{g}}\left(c_{s}^{p} c_{p}^{s}-\left(S_{p}^{\prime}\left(c_{p}^{s} \varphi(x)+u_{p}^{\star}\right)\right)^{-1} T_{s}^{\prime}(\varphi(x))\right)\left(c_{g}^{g}+T_{g}^{\prime}(x)\right)-1
$$

Using again the chain rule to compute $\left(S_{p}^{\prime}\left(c_{p}^{s} \varphi(x)+u_{p}^{\star}\right)\right)^{-1}$ leads to

$$
L^{\prime}(x)=\frac{S_{p}^{\prime}\left(c_{p}^{s} \varphi(x)+u_{p}^{\star}\right)}{c_{g}^{s} c_{s}^{g}}\left(c_{s}^{p} c_{p}^{s}-T_{s}^{\prime}(\varphi(x)) T_{p}^{\prime}\left(S_{p}\left(c_{p}^{s} \varphi(x)+u_{p}^{\star}\right)\right)\right)\left(c_{g}^{g}+T_{g}^{\prime}(x)\right)-1 .
$$

By Assumption 1, this function is continuous at each point for which it is defined.

Claim 1 For a given $u_{g}^{\star}$, assume that there exists $x_{g}^{\star}$ such that $L^{\prime}\left(x_{g}^{\star}\right)>0$. Then, for every $u_{p}^{\star}$ there exists $u_{s}^{\star}$ such that the system admits at least three distinct equilibria.

Proof of Claim 1 On the one hand, since $L^{\prime}\left(x_{g}^{\star}\right)>0$ and $L^{\prime}$ is continuous, there exists a strictly positive real number $\epsilon_{0}$ such that $x_{g}^{*} \in\left(x_{g}^{0}+\epsilon_{0}, x_{g}^{1}-\epsilon_{0}\right)$ and $L^{\prime}(x)>0$ for each $x \in\left(x_{g}^{0}+\epsilon_{0}, x_{g}^{1}-\epsilon_{0}\right)$. Additionally, for every $u_{p}^{\star}$ we can chose $u_{s}^{\star}$ such that $L\left(x_{g}^{\star}\right)=0$. Hence, we can assume that $L\left(x_{g}^{0}+\epsilon_{0}\right)<0$ and $L\left(x_{g}^{0}-\epsilon_{0}\right)>0$. But, on the other hand, it follows from (31) that there exist $\epsilon_{1}<\epsilon_{0}$ such that $L(x)>0$ over $\left(x_{g}^{0}, x_{g}^{0}+\epsilon_{1}\right)$ and $L(x)<0$ over $\left(x_{g}^{1}, x_{g}^{1}-\epsilon_{1}\right)$. Therefore, by the continuity of $L$ and by the intermediate value theorem, there exist two points $x_{1}^{\star}$ and $x_{2}^{\star}$ such that $x_{1}^{\star} \in\left(x_{g}^{0}+\epsilon_{1}, x_{g}^{0}+\epsilon_{0}\right)$ and $x_{2}^{\star} \in\left(x_{g}^{1}-\epsilon_{0}, x_{g}^{1}-\epsilon_{1}\right)$, and that satisfy, respectively, the equations $L\left(x_{1}^{\star}\right)=0$ and $L\left(x_{2}^{\star}\right)=0$.

Fourth step. Using the previous result, we can continue with the proof of Theorem 1 and treat, one after the other, its three cases.

Item (i). The first case appears when $\sigma_{p} \sigma_{s} c_{p}^{s} c_{s}^{p} \leq 1$. In this case $c_{p}^{s} c_{s}^{p}-T_{p}^{\prime}\left(S_{p}\left(c_{p}^{s} \varphi(x)+u_{p}^{\star}\right)\right) T_{s}^{\prime}(\varphi(x)) \leq 0$ because that the infinimum of the function $T_{p}^{\prime} T_{s}^{\prime}$ is equal to $1 / \sigma_{p} \sigma_{s}$. Since $c_{g}^{g}+T_{g}^{\prime}(x) \geq 1 / \sigma_{g} \geq 0$, we must have in view of $(32) L^{\prime}(x)<0$, for each $x \in\left(x_{g}^{0}, x_{g}^{1}\right)$. The uniqueness of the equilibrium point comes from the fact that $L$ is strictly decreasing. Its existence is guaranteed by the intermediate value theorem and by the limits of $L$ at each end of its domain (31).

Item (ii). In this case it must be shown that, when $\sigma_{p} \sigma_{s} c_{p}^{s} c_{s}^{p}>1$, there always exist $u_{g}^{\star}$ and a point $x^{\star}$ such that $L^{\prime}\left(x^{\star}\right)>0$. To this end, for a given $u_{g}^{\star}$, we look for a point $x^{\star}$ such that $T_{s}^{\prime}\left(\varphi\left(x^{\star}\right)\right) T_{p}^{\prime}\left(c_{p}^{s} \varphi\left(x^{\star}\right)+\right.$ $\left.u_{p}^{\star}\right)=1 / \sigma_{p} \sigma_{s}$. We know from the Assumption 1 That there exist a point $y^{\star}$ where $T_{s}$ reaches its minimum $1 / \sigma_{s}$. For this $y^{\star}$ and for every $u_{g}^{\star}$ there exist a point $x^{\star}$, such that $\varphi\left(x^{\star}\right)=y^{\star}$. The existence of $x^{\star}$ comes from the injectivity of $\varphi$. In other words, the equation

$$
\frac{1}{c_{g}^{s}}\left(c_{g}^{g} x+T_{g}(x)+u_{g}^{\star}\right)=y^{\star}
$$

always admits a unique solution $x^{\star}$ in $\left(x_{g}^{0}, x_{g}^{1}\right)$. Furthermore, since the map $x_{s} \mapsto c_{p}^{s} x_{s}+u_{p}$ is bijective, then there exists a constant input $u_{p}^{\star}$ such that $T_{p}^{\prime}\left(c_{p}^{s} x_{s}^{\star}+u_{p}^{\star}\right)=1 / \sigma_{p}$. Moreover, applying $S_{g}$ to both sides of (33), we obtain

$$
\lim _{u_{g}^{\star} \rightarrow+\infty} x^{\star}=S_{g}\left(c_{g}^{s} y^{\star}-c_{g}^{g} x^{\star}-u_{g}^{\star}\right)=1 .
$$

Now, combining the equality

$$
c_{s}^{p} c_{p}^{s}-T_{s}^{\prime}\left(\varphi\left(x^{\star}\right)\right) T_{p}^{\prime}\left(c_{p}^{s} \varphi\left(x^{\star}\right)+u_{p}^{\star}\right)=c_{s}^{p} c_{p}^{s}-1 / \sigma_{p} \sigma_{s}
$$

with the limit

$$
\lim _{u_{g}^{\star} \rightarrow+\infty} c_{g}^{g}+T_{g}^{\prime}\left(x^{\star}\right)=+\infty
$$

we have, for $u_{g}^{\star}$ big enough, that

$$
\left(c_{s}^{p} c_{p}^{s}-T_{s}^{\prime}\left(\varphi\left(x^{\star}\right)\right) T_{p}^{\prime}\left(c_{p}^{s} \varphi\left(x^{\star}\right)+u_{p}^{\star}\right)\right)\left(c_{g}^{g}+T_{g}^{\prime}\left(x^{\star}\right)\right)>\frac{c_{g}^{s} c_{s}^{g}}{\sigma_{p}} .
$$


For such an $u_{g}^{\star}$, we have $L^{\prime}\left(x^{\star}\right)>0$. Hence, by Claim 1, there exists $u_{s}^{\star}$ such that the dynamics (1) has at least three distinct equilibria.

Item (iii). For the third case, observe that the relation $\left(\sigma_{p} c_{p}^{s} c_{s}^{p}-\frac{1}{\sigma_{s}}\right)\left(c_{g}^{g}+\frac{1}{\sigma_{g}}\right)>c_{g}^{s} c_{s}^{g}$ is satisfied if and only if

$$
c_{p}^{s} c_{s}^{p}>\frac{1}{\sigma_{p} \sigma_{s}}+\frac{c_{g}^{s} c_{s}^{g}}{c_{g}^{g}+\frac{1}{\sigma_{g}}} .
$$

Therefore, the condition imposed by this case is more restrictive than that of the previous one. The key point of the proof is to be able to find, for each fixed $u_{g}^{\star}$, a point $x^{\star}$ such that $L^{\prime}\left(x^{\star}\right)>0$. On the one hand, we have

$$
c_{g}^{g}+T_{g}^{\prime}(x) \geq c_{g}^{g}+\frac{1}{\sigma_{g}},
$$

for each $x \in(0,1)$. On the other hand, by Assumption 1, there is always a point on which the function $x \mapsto T_{s}^{\prime}(x) T_{p}^{\prime}\left(S_{p}\left(c_{p}^{s} x+u_{p}^{\star}\right)\right)$ reaches its minimum $\sigma_{p} \sigma_{s}$. Define $y^{\star}$ to be this point. Now, take the only $x^{\star}$ such that $\varphi\left(x^{\star}, u_{g}^{\star}\right)=y^{\star}$. This is always possible because $\varphi$ is both surjective and strictly increasing, for each $u_{i}^{\star}$. Hence, in view of $(32)$,

$$
\begin{aligned}
L^{\prime}\left(x^{\star}\right) & =\frac{\sigma_{p}}{c_{g}^{s} c_{s}^{g}}\left(c_{p}^{s} c_{s}^{p}-\frac{1}{\sigma_{p} \sigma_{s}}\right)\left(c_{g}^{g}+T_{g}^{\prime}\left(x^{\star}\right)\right)-1 \\
& \geq \frac{\sigma_{p}}{c_{g}^{s} c_{s}^{g}}\left(c_{p}^{s} c_{s}^{p}-\frac{1}{\sigma_{p} \sigma_{s}}\right)\left(c_{g}^{g}+\frac{1}{\sigma_{g}}\right)-1 \\
& >0
\end{aligned}
$$

Since $L^{\prime}\left(x^{\star}\right)>0$, the proof of this last case of the theorem follows from Claim 1 .

Proof of Proposition 1 Item (i). The Jacobian matrix of dynamics 1 at the equilibrium $x^{\star}=\left(x_{s}^{\star}, x_{g}^{\star}, x_{p}^{\star}\right)^{T}$ is given by:

$$
J\left(x^{\star}\right):=\left(\begin{array}{ccc}
-\frac{1}{\tau_{s}} & -\frac{c_{s}^{g}}{\tau_{s}} \sigma_{s}^{\star} & \frac{c_{s}^{p}}{\tau_{s}} \sigma_{s}^{\star} \\
\frac{c_{g}^{s}}{\tau_{g}} \sigma_{g}^{\star} & -\frac{1}{\tau_{g}}-\frac{c_{g}^{g}}{\tau_{g}} \sigma_{g}^{\star} & 0 \\
\frac{c_{p}^{s}}{\tau_{p}} \sigma_{p}^{\star} & 0 & -\frac{1}{\tau_{p}}
\end{array}\right) .
$$

Its characteristic polynomial is given by

$$
P_{J}(\lambda)=\lambda^{3}+a_{1} \lambda^{2}+a_{2} \lambda+a_{3}
$$

where

$$
\begin{gathered}
a_{1}=\frac{1}{\tau_{s}}+\frac{1}{\tau_{p}}+\frac{1}{\tau_{g}}\left(1+c_{g}^{g} \sigma_{g}^{\star}\right) \\
a_{2}=\frac{1}{\tau_{g}}\left(\frac{1}{\tau_{s}}+\frac{1}{\tau_{p}}\right)\left(1+c_{g}^{g} \sigma_{g}^{\star}\right)+\frac{1}{\tau_{s}} \frac{1}{\tau_{p}}\left(1-c_{s}^{p} c_{p}^{s} \sigma_{s}^{\star} \sigma_{p}^{\star}\right)+\frac{1}{\tau_{s}} \frac{1}{\tau_{g}} c_{s}^{g} c_{g}^{s} \sigma_{s}^{\star} \sigma_{g}^{\star}
\end{gathered}
$$

and

$$
a_{3}=\frac{1}{\tau_{s}} \frac{1}{\tau_{g}} \frac{1}{\tau_{p}}\left[\left(1+c_{g}^{g} \sigma_{g}^{\star}\right)\left(1-c_{s}^{p} c_{p}^{s} \sigma_{s}^{\star} \sigma_{p}^{\star}\right)+c_{s}^{g} c_{g}^{s} \sigma_{s}^{\star} \sigma_{g}^{\star}\right]
$$

According to the Routh-Hurwitz criterion, if $a_{1}>0, a_{3}>0$ and $a_{1} a_{2}-a_{3}>0$, then all the eigenvalues of $J$ have negative real part. We have clearly $a_{1}>0$. Furthermore, we have $a_{3}>0$ if and only if (7) holds. For the sign of $a_{1} a_{2}-a_{3}$, we make use of the following notations

$$
e_{1}=1-c_{s}^{p} c_{p}^{s} \sigma_{s}^{\star} \sigma_{p}^{\star}, e_{2}=1+c_{g}^{g} \sigma_{g}^{\star} \text { and } e_{3}=c_{s}^{g} c_{g}^{s} \sigma_{s}^{\star} \sigma_{g}^{\star}
$$


Using this notations, one obtains

$$
\begin{aligned}
a_{1} a_{2}-a_{3}= & \left(\frac{1}{\tau_{s}}+\frac{1}{\tau_{p}}\right)^{2} \frac{1}{\tau_{g}} e_{2}+\left(\frac{1}{\tau_{s}}+\frac{1}{\tau_{p}}\right) \frac{1}{\tau_{s}} \frac{1}{\tau_{p}} e_{1}+\left(\frac{1}{\tau_{s}}+\frac{1}{\tau_{p}}\right)\left(\frac{1}{\tau_{g}}\right)^{2} e_{2}^{2}+\frac{1}{\tau_{g}}\left(\frac{1}{\tau_{s}}\right)^{2} e_{3}+\frac{1}{\tau_{s}}\left(\frac{1}{\tau_{g}}\right)^{2} e_{2} e_{3} \\
= & \frac{\tau_{s}+\tau_{p}}{\tau_{s}^{2} \tau_{p}^{2}}\left(\frac{\tau_{s}+\tau_{p}}{\tau_{g}} e_{2}+e_{1}+\frac{\tau_{s} \tau_{p}}{\tau_{g}^{2}} e_{2}^{2}+\frac{\tau_{p}^{2}}{\tau_{g}\left(\tau_{s}+\tau_{p}\right)} e_{3}+\frac{\tau_{s} \tau_{p}^{2}}{\tau_{g}^{2}\left(\tau_{s}+\tau_{p}\right)} e_{2} e_{3}\right) \\
= & \frac{\tau_{s}+\tau_{p}}{\tau_{s}^{2} \tau_{p}^{2}}\left(1+\frac{\tau_{s}+\tau_{p}}{\tau_{g}}\left(1+c_{g}^{g} \sigma_{g}^{\star}\right)+\frac{\tau_{s} \tau_{p}}{\tau_{g}^{2}}\left(1+c_{g}^{g} \sigma_{g}^{\star}\right)^{2}+\frac{\tau_{p}^{2}}{\tau_{g}\left(\tau_{s}+\tau_{p}\right)} c_{s}^{g} c_{g}^{s} \sigma_{s}^{\star} \sigma_{g}^{\star}\right. \\
& \left.+\frac{\tau_{s} \tau_{p}^{2}}{\tau_{g}^{2}\left(\tau_{s}+\tau_{p}\right)}\left(1+c_{g}^{g} \sigma_{g}^{\star}\right) c_{s}^{g} c_{g}^{s} \sigma_{s}^{\star} \sigma_{g}^{\star}-c_{s}^{p} c_{p}^{s} \sigma_{s}^{\star} \sigma_{p}^{\star}\right) .
\end{aligned}
$$

If (8) holds then we have $a_{1} a_{2}-a_{3}>0$. Hence, if (7) and (8) holds, the Jacobian matrix (34) is Hurwitz, which establishes that $x^{\star}$ is locally exponentially stable.

Item (ii). With a slight abuse of notation, we define $f_{i}=\left(-x_{i}+S_{i}\right) / \tau_{i}$ for each $i \in\{s, g, p\}$. That is, we omit the arguments of these two functions, which leads to $\dot{x}_{i}=f_{i}$. Now, define the function $V: \mathbb{R}^{3} \rightarrow \mathbb{R}$ given by

$$
V(x)=\frac{1}{2} \sum_{i=s, g, p} \tau_{i} f_{i}^{2}(x) .
$$

The first part of condition (9) implies, by Theorem 1, the uniqueness of the equilibrium point of system (1), which is then the only point for which

$$
V\left(x^{\star}\right)=0
$$

Furthermore, the derivative of $V$ along (1) yield,

$$
\begin{aligned}
\dot{V} & =\frac{\partial V}{\partial x_{s}} f_{s}+\frac{\partial V}{\partial x_{g}} f_{g}+\frac{\partial V}{\partial x_{p}} f_{p} \\
& =-f_{s}^{2}-\left(1+c_{g}^{g} S_{g}^{\prime}\right) f_{g}^{2}-f_{p}^{2}+\left(c_{g}^{s} S_{g}^{\prime}-c_{s}^{g} S_{s}^{\prime}\right) f_{s} f_{g}+\left(c_{p}^{s} S_{p}^{\prime}+c_{s}^{p} S_{s}^{\prime}\right) f_{s} f_{p} \\
& <-f_{s}^{2}-f_{g}^{2}-f_{p}^{2}+\left(c_{g}^{s} S_{g}^{\prime}-c_{s}^{g} S_{s}^{\prime}\right) f_{s} f_{g}+\left(c_{p}^{s} S_{p}^{\prime}+c_{s}^{p} S_{s}^{\prime}\right) f_{s} f_{p}
\end{aligned}
$$

Using Young's inequality, we obtain

$$
\begin{aligned}
\dot{V} & <-f_{s}^{2}-f_{g}^{2}-f_{p}^{2}+\frac{1}{2}\left(c_{g}^{s} S_{g}^{\prime}+c_{s}^{g} S_{s}^{\prime}\right)\left(f_{s}^{2}+f_{g}^{2}\right)+\frac{1}{2}\left(c_{p}^{s} S_{p}^{\prime}+c_{s}^{p} S_{s}^{\prime}\right)\left(f_{s}^{2}+f_{p}^{2}\right) \\
& \leq-f_{s}^{2}-f_{g}^{2}-f_{p}^{2}+\frac{1}{2}\left(c_{g}^{s} \sigma_{g}+c_{s}^{g} \sigma_{s}\right)\left(f_{s}^{2}+f_{g}^{2}\right)+\frac{1}{2}\left(c_{p}^{s} \sigma_{p}+c_{s}^{p} \sigma_{s}\right)\left(f_{s}^{2}+f_{p}^{2}\right) \\
& <\left(-1+\frac{1}{2}\left(c_{g}^{s} \sigma_{g}+c_{s}^{g} \sigma_{s}\right)+\frac{1}{2}\left(c_{p}^{s} \sigma_{p}+c_{s}^{p} \sigma_{s}\right)\right)\left(f_{s}^{2}+f_{g}^{2}+f_{p}^{2}\right) .
\end{aligned}
$$

Therefore, when the second part of condition (9) is satisfied, we can always find $\epsilon>0$ such that

$$
\dot{V} \leq-\epsilon\left(f_{s}^{2}+f_{g}^{2}+f_{p}^{2}\right)
$$

and then

$$
\dot{V} \leq-k V
$$

where $k=\frac{2 \epsilon}{\max _{i}\left(\tau_{i}\right)}$.

Now, by Theorem 1, the condition (7) implies the uniqueness of the equilibrium point, which is then the only point for which $V(x)=0$. It follows that $V$ is a Lyapunov function that satisfies the standard conditions for global asymptotic stability [11, Theorem 4.1]. 
Proof of Proposition 2 Suppose that for every $\left(u_{s}^{\star}, u_{g}^{\star}, u_{p}^{\star}\right) \in \mathbb{R}^{3}$ system (1) has a unique equilibrium point. This implies (from Theorem 1) that (2) holds. If $c_{s}^{s} \leq \sigma_{p} c_{s}^{p} c_{p}^{s}$ then, under Assumption 1 and precisely the fact that $\sigma_{s}>0$, we have

$$
\sigma_{s} c_{s}^{s} \leq \sigma_{p} \sigma_{s} c_{s}^{p} c_{p}^{s}
$$

Thus, we have

$$
\sigma_{s} c_{s}^{s} \leq 1
$$

From [18, Theorem 1], (38) implies the existence and uniqueness of equilibrium to system (11). Suppose now that for every $\left(u_{s}^{\star}, u_{g}^{\star}\right) \in \mathbb{R}^{3}$ system (11) has a unique equilibrium point. This implies (from [18, Theorem 1]) that (38) holds. If

$$
c_{s}^{s} \geq \sigma_{p} c_{s}^{p} c_{p}^{s}
$$

then we obtain the following inequalities

$$
\sigma_{p} \sigma_{s} c_{s}^{p} c_{p}^{s} \leq 1
$$

which implies (from Theorem 1) the existence and uniqueness of equilibrium to system (1).

Proof of Proposition 3 If $\sigma_{s} c_{s}^{s} \leq 1$ and $\sigma_{p} \sigma_{s} c_{s}^{p} c_{p}^{s} \leq 1$ then system (1) and (11) has a unique equilibrium point in D, respectively. Suppose that the condition (10) is satisfied, which means that the equilibrium point of (1) is globally asymptotically stable. If condition (12) is satisfied, then one can easily verify that we have

$$
\frac{1}{2}\left(\sigma_{s} c_{s}^{g}+\sigma_{g} c_{g}^{s}\right)<1-\sigma_{s} c_{s}^{s} .
$$

From [18, Proposition 1], (39) implies the global asymptotic stability of the equilibrium point of (11).

\subsection{Proof of Theorem 2}

Proof of Proposition 4 Notice that, for any $\alpha>0, s \mapsto e^{-\alpha s}$ belongs to $\hat{\mathcal{A}}_{-}$(defined in section A.1) and so all the components of $K$ are in $\hat{\mathcal{A}}_{-}$. For the transfer matrix $G$, the functions $H_{s}$ and $H_{p}$ are strictly proper, rational transfer functions with one pole at $s=-1 / \tau_{s}$ and $s=-1 / \tau_{p}$ respectively. Clearly, $H_{s}$ and $H_{p}$ belong to $\hat{\mathcal{A}}_{-}$. In fact, $H_{s}$ and $H_{p}$ are the Laplace transform of

$$
h_{s}(t):=\frac{\sigma_{s}^{\star}}{\tau_{s}} e^{-\frac{1}{\tau_{s}} t} \quad \text { and } \quad h_{p}(t):=\frac{\sigma_{p}^{\star}}{\tau_{s}} e^{-\frac{1}{\tau_{p}} t}
$$

respectively. Then, one can observe that $e^{\epsilon t} h_{s}(t)$ and $e^{\epsilon t} h_{p}(t)$ belong to $L_{1}(0, \infty)$ for every $\epsilon$ in the interval $\left(0, \min \left(1 / \tau_{s}, 1 / \tau_{p}\right)\right)$. This is not the case of $H_{g}$. Indeed, $H_{g}$ belong to $\hat{\mathcal{B}}$ (defined in section A.1). In fact, up to multiplication by a constant, the transfer function $H_{g}$ has the following form

$$
H(s)=\frac{1}{a s+b+c e^{-d s}}
$$

where $a, b, c$ and $d \in \mathbb{R}$. It is proved [5, Example 7.1.12, p. 343] that every transfer function having this form is an element of $\hat{\mathcal{B}}$.

Proof of Proposition 5 To establish Proposition 5 we invoke the Nyquist Theorem (given by Theorem 3 in Section A.2). To that aim, we need to evaluate $p_{G}$ and $p_{K}$, the number of poles of $G$ and $K$ in $\overline{\mathbb{C}_{0}^{+}}$(see Section A for the definition of $\overline{\mathbb{C}_{\beta}^{+}}$), and then check if the matrix $G K$ is strictly proper on $\overline{\mathbb{C}_{0}^{+}}$. Concerning the first point, we know that under the condition of stability of $H_{g}$, the two transfer matrices $G$ and $K$ belong to $\mathcal{M} \hat{\mathcal{A}}_{-}$(defined in Section A.1) and thus they have no poles in $\overline{\mathbb{C}_{0}^{+}}$, which means that $p_{G}=p_{K}=0$. For the second point, we recall (see Definition 3 in Section A.2) that a transfer matrix $M$ is strictly proper on $\overline{\mathbb{C}_{0}^{+}}$if and only if

$$
\lim _{\rho \rightarrow \infty} \sup _{s \in \overline{\mathbb{C}}_{0}^{+},|s| \geq \rho}|M(s)|=0,
$$


where the norm |.| denote a matrix norm. We have

$$
G(s) K(s)=\left(\begin{array}{ccc}
0 & -c_{s}^{g} e^{-\delta_{s}^{g} s} H_{s}(s) & c_{s}^{p} e^{-\delta_{s}^{p} s} H_{s}(s) \\
c_{g}^{s} e^{-\delta_{g}^{s} s} H_{g}(s) & 0 & 0 \\
c_{p}^{s} e^{-\delta_{p}^{s} s} H_{p}(s) & 0 & 0
\end{array}\right) .
$$

It is obvious that $G K$ is strictly proper on $\overline{\mathbb{C}_{0}^{+}}$since all its components are strictly proper on $\overline{\mathbb{C}_{0}^{+}}$. Consequently, by straightforward application of Nyquist Theorem, we deduce, under the condition of stability of $H_{g}$, that the feedback system defined by (15) is input-output stable if and only if

$$
\operatorname{det}(I-G K)=0 .
$$

Remark that one can easily obtain the following equality

$$
\operatorname{det}(I-G K)=1-H_{s}\left(K_{p}+K_{g}\right)
$$

which concludes the proof of Proposition 5.

Proof of Lemma 2 First step. We start by proving that the transfer functions $H_{s p}$ and $H_{s g}$, introduced by (24), are well defined in $\hat{\mathcal{A}}_{-}$. To that aim, notice that the two transfer functions $H_{s}$ and $K_{p}$ belong

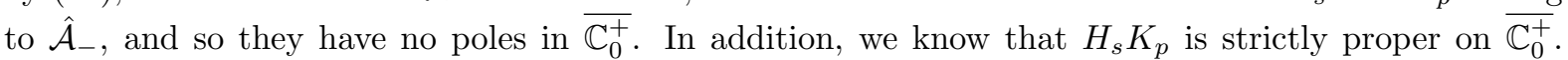
Consequently, under the assumption that the feedback $\left(H_{s}, K_{p}\right)$ is input-output stable, Nyquist Theorem (see Theorem 3 in Section A.2) implies that we have ind $\left(1-H_{s} K_{p}\right)=0$. And so, by Principle of the Argument [5, Theorem A.1.13, p.569], we have that $1-H_{s} K_{p}$ has no zeros in $\overline{\mathbb{C}_{0}^{+}}$. Then $1-H_{s} K_{p}$ is invertible over $\hat{\mathcal{A}}_{-}$(cf. Section A.1 (property $\left.\left(p_{3}\right)\right)$ ). Hence, the transfer function $H_{s p}$ is well defined in $\hat{\mathcal{A}}_{-}$. The same reasoning holds for the transfer function $H_{s g}$.

Second step. From the fact that $1-H_{s} K_{p}$ is invertible over $\hat{\mathcal{A}}_{-}$, the following equality

$$
\begin{aligned}
1-H_{s p} K_{g} & =1-\frac{H_{s} K_{g}}{1-H_{s} K_{p}} \\
& =\frac{1-H_{s}\left(K_{p}+K_{g}\right)}{1-H_{s} K_{p}},
\end{aligned}
$$

which can be written, equivalently in $\hat{\mathcal{A}}_{-}$, as the following

$$
\left(1-H_{s p} K_{g}\right)\left(1-H_{s} K_{p}\right)=1-H_{s}\left(K_{p}+K_{g}\right),
$$

is well defined in $\hat{\mathcal{A}}_{-}$.

Third step. We now prove that the transfer function $1-H_{s}\left(K_{p}+K_{g}\right)$ has a well defined Nyquist index. To proof this, we recall that a scalar complex-valued function $F$ defined and meromorphic on $\mathbb{C}_{-\epsilon}^{+}$(see Section A for the definition of $\mathbb{C}_{-\epsilon}^{+}$) for some $\epsilon>0$ has a well defined Nyquist index [5, Definition A.1.15, p. 569] if it has a nonzero limit at infinity in $\overline{\mathbb{C}_{0}^{+}}$; this means (see Definition 3 in Section A.2) that

$$
\lim _{\rho \rightarrow \infty}\left[\sup _{s \in \overline{\mathbb{C}_{0}^{+}}|| s \mid \geq \rho}|F(s)-F(\infty)|\right]=0
$$

for some nonzero $F(\infty) \in \mathbb{C}$. Take $F(s)=1-H_{s}(s)\left(K_{p}(s)+K_{g}(s)\right)$. Since $H_{s}, K_{p}, K_{g}$ belong to $\hat{\mathcal{A}}_{-}$, we have that $F$ belongs to $\hat{\mathcal{A}}_{-}$. Hence, it is holomorphic on $\mathbb{C}_{-\epsilon}^{+}$for some $\epsilon>0$ [5, Lemma 7.1.5, p. 338]. Moreover, we know that $H_{s}\left(K_{p}+K_{g}\right)$ is strictly proper on $\overline{\mathbb{C}_{0}^{+}}$, hence we have that $F(\infty)=1$. We conclude that $1-H_{s}\left(K_{p}+K_{g}\right)$ has a well defined Nyquist index. Similarly, $1-H_{s p} K_{g}$ has a well defined Nyquist index. 
Fourth step. We are now able to evoke some properties of the Nyquist index like the following [5, Equation (A.1.9), p. 570]

$$
\operatorname{ind}\left(F_{1} F_{2}\right)=\operatorname{ind}\left(F_{1}\right)+\operatorname{ind}\left(F_{2}\right) .
$$

Using this property, we obtain

$$
\operatorname{ind}\left(1-H_{s p} K_{g}\right)+\operatorname{ind}\left(1-H_{s} K_{p}\right)=\operatorname{ind}\left(1-H_{s}\left(K_{p}+K_{g}\right)\right) .
$$

Together with the fact that ind $\left(1-H_{s} K_{p}\right)=0$, this implies that

$$
\operatorname{ind}\left(1-H_{s p} K_{g}\right)=\operatorname{ind}\left(1-H_{s}\left(K_{p}+K_{g}\right)\right) .
$$

In the same way one can show that we also have

$$
\operatorname{ind}\left(1-H_{s g} K_{p}\right)=\operatorname{ind}\left(1-H_{s}\left(K_{p}+K_{g}\right)\right),
$$

which ends the proof of Lemma 2.

Proof of Lemma 3 In view of (24) the transfer function $H_{s p}$ is given by

$$
H_{s p}=\frac{H_{s}}{1-H_{s} K_{p}} .
$$

By replacing $H_{s}$ and $K_{p}$ by their expressions (13)-(21), we obtain

$$
H_{s p}(s)=\sigma_{s}^{\star} \frac{1+\tau_{p} s}{\left(1+\tau_{p} s\right)\left(1+\tau_{s} s\right)-c_{p} \sigma_{s}^{\star} \sigma_{p}^{\star} e^{-\delta_{p} s}} .
$$

From (18), the loop gain $\gamma_{H_{s p}}$ of $H_{s p}$ is given by:

$$
\gamma_{H_{s p}}(w)=20 \log \left(\left|H_{s p}(i w)\right|\right) .
$$

One can easily verify that we have

$$
\left|H_{s p}(i w)\right|=\sigma_{s}^{\star}\left(\frac{1+\tau_{p}^{2} w^{2}}{\left(1-\tau_{b} w^{2}-c_{a} \cos \left(\delta_{p} w\right)\right)^{2}+\left(\tau_{a} w+c_{a} \sin \left(\delta_{p} w\right)\right)^{2}}\right)^{1 / 2}
$$

where

$$
c_{a}:=c_{p} \sigma_{s}^{\star} \sigma_{p}^{\star}, \tau_{b}:=\tau_{s} \tau_{p} \text {, and } \tau_{a}:=\tau_{s}+\tau_{p} .
$$

Let $f(\cdot)$ be the following function

$$
f(w):=\left(\frac{\gamma_{H_{s p}}(i w)}{\sigma_{s}^{\star}}\right)^{2} .
$$

After developing the denominator of $f(w)$, we obtain

$$
f(w)=\underbrace{\frac{1+\tau_{p}^{2} w^{2}}{\left(1-\tau_{b} w^{2}\right)^{2}+\tau_{a}^{2} w^{2}+c_{a}^{2}}+\underbrace{2 c_{a} \tau_{a} \sin \left(\delta_{p} w\right) w-2 c_{a}\left(1-\tau_{b} w^{2}\right) \cos \left(\delta_{p} w\right)}_{F_{2}(w)} .}_{F_{1}(w)}
$$

The derivative of $f(\cdot)$ with respect to $w$ is given by:

$$
f^{\prime}(w)=\frac{2 \tau_{p}^{2} w\left(F_{1}(w)+F_{2}(w)\right)-\left(1+\tau_{p}^{2} w^{2}\right)\left(F_{1}^{\prime}(w)+F_{2}^{\prime}(w)\right)}{\left(F_{1}(w)+F_{2}(w)\right)^{2}} .
$$

The derivative of $F_{1}(w)$ is given by:

$$
\begin{aligned}
F_{1}^{\prime}(w) & =-4 \tau_{b} w\left(1-\tau_{b} w^{2}\right)+2 \tau_{a}^{2} w \\
& =4 \tau_{b}^{2} w^{3}+2\left(\tau_{s}^{2}+\tau_{p}^{2}\right) w .
\end{aligned}
$$


Consequently, one can verify that we have

$$
2 \tau_{p}^{2} w F_{1}(w)-\left(1+\tau_{p}^{2} w^{2}\right) F_{1}^{\prime}(w)=-2 \tau_{p}^{2} \tau_{b}^{2} w^{5}-4 \tau_{b}^{2} w^{3}+2\left(c_{a}^{2} \tau_{p}^{2}-\tau_{s}^{2}\right) w .
$$

In the same way, the derivative of $F_{2}$ is given by:

$$
\begin{aligned}
F_{2}^{\prime}(w) & =4 c_{a} \tau_{b} \cos \left(\delta_{p} w\right) w+2 c_{a}\left(1-\tau_{b} w^{2}\right) \delta_{p} \sin \left(\delta_{p} w\right)+2 c_{a} \tau_{a} \sin \left(\delta_{p} w\right)+2 c_{a} \tau_{a} \delta_{p} \cos \left(\delta_{p} w\right) w \\
& =2 c_{a}\left(2 \tau_{b}+\tau_{a} \delta_{p}\right) \cos \left(\delta_{p} w\right) w+2 c_{a}\left(\tau_{a}+\left(1-\tau_{b} w^{2}\right) \delta_{p}\right) \sin \left(\delta_{p} w\right) .
\end{aligned}
$$

It follows that

$$
\begin{aligned}
2 \tau_{p}^{2} w F_{2}(w)-\left(1+\tau_{p}^{2} w^{2}\right) F_{2}^{\prime}(w) & =\left(-4 \tau_{p}^{2} c_{a}-4 \tau_{b} c_{a}-2 c_{a} \tau_{a} \delta_{p}\left(1+\tau_{p}^{2} w^{2}\right)\right) \cos \left(\delta_{p} w\right) w \\
& +\left(2 \tau_{p}^{2} \tau_{a} c_{a} w^{2}-2 \tau_{a} c_{a}-2 c_{a} \delta_{p}\left(1+\tau_{p}^{2} w^{2}\right)\left(1-\tau_{b} w^{2}\right)\right) \sin \left(\delta_{p} w\right) .
\end{aligned}
$$

Using the fact that, for all $w \in \mathbb{R}$, we have the following properties:

$$
-1 \leq \cos (w) \leq 1 \quad \text { and }-w \leq \sin (w) \leq w,
$$

the quantity $2 \tau_{p}^{2} w F_{2}(w)-\left(1+\tau_{p}^{2} w^{2}\right) F_{2}^{\prime}(w)$ can be bounded as follows

$$
\begin{aligned}
2 \tau_{p}^{2} w F_{2}(w)-\left(1+\tau_{p}^{2} w^{2}\right) F_{2}^{\prime}(w) & \leq\left(4 \tau_{p}^{2} c_{a}+4 \tau_{b} c_{a}+4 \tau_{a} c_{a} \delta_{p}+2 c_{a} \delta_{p}^{2}\right) w \\
& +\left(4 \tau_{a} \tau_{p}^{2} c_{a} \delta_{p}+2 \tau_{p}^{2} c_{a} \delta_{p}^{2}+2 c_{a} \tau_{b} \delta_{p}^{2}\right) w^{3} \\
& +2 c_{a} \tau_{b} \tau_{p}^{2} \delta_{p}^{2} w^{5} .
\end{aligned}
$$

Therefore, by regrouping the terms of $f^{\prime}(w)$, one obtain the following inequality

$$
\frac{\left(F_{1}(w)+F_{2}(w)\right)^{2}}{2 w} f^{\prime}(w) \leq F_{3}\left(w^{2}\right),
$$

where $F_{3}$ is given by the following polynomial expression

$$
\begin{aligned}
F_{3}(x) & =\underbrace{\tau_{b} \tau_{p}^{2}\left(\delta_{p}^{2} c_{a}-\tau_{b}\right)}_{\alpha_{1}} x^{2} \\
& +\underbrace{\left(\left(\left(\tau_{p}^{2}+\tau_{b}\right) \delta_{p}^{2}+2 \tau_{a} \tau_{p}^{2} \delta_{p}\right) c_{a}-2 \tau_{b}^{2}\right)}_{\alpha_{2}} x \\
& +\underbrace{\tau_{p}^{2} c_{a}^{2}+\left(\delta_{p}^{2}+2 \tau_{a} \delta_{p}+2 \tau_{p}^{2}+2 \tau_{b}\right) c_{a}-\tau_{s}^{2}}_{\alpha_{3}} .
\end{aligned}
$$

Remark that if $\alpha_{1}, \alpha_{2}$ and $\alpha_{3}$ are strictly less than zero then the quantity $F_{3}\left(w^{2}\right)$ is negative for every positive real number $w$. And thus, in view of (41), the derivative of $\left|H_{s p}(i w)\right|$ is negative on $\mathbb{R}^{+}$. In view of (40), one can easily check that for each fixed $\delta_{p}>0$, one can choose $c_{p}\left(\delta_{p}\right)>0$ for which $\alpha_{1}, \alpha_{2}$ and $\alpha_{3}$ are strictly less than zero. Hence, for each $\delta_{p}>0$, there exist $c_{p}^{\star}\left(\delta_{p}\right)>0$ for which the loop gain $\gamma_{H_{s p}}$ is strictly decreasing for every $c_{p} \in\left(0, c_{p}^{\star}\left(\delta_{p}\right)\right)$.

Proof of Lemma 4 For item $(i)$, knowing that the two transfer functions $H_{s}$ and $H_{p}$ are input-output stable and their gains are strictly decreasing, then by direct application of [18, Theorem 2] we have that $H_{s p}$ is input-output stable if and only if $\delta_{p}<\Delta\left(G_{s p}\right)$. When $c_{p} \sigma_{p}^{\star} \sigma_{s}^{\star}<1$, the stability of $H_{s p}$ results from the small-gain theorem [5, Theorem 9.1.7] and does not depend on the value of $\delta_{p} \geq 0$. In this case we have $\Delta\left(G_{s p}\right)=+\infty$. This case is illustrated in Figure 5 A. While, when $c_{p} \sigma_{p}^{\star} \sigma_{s}^{\star} \geq 1$, it is easy to show that the Nyquist locus of $G_{s p}$ encircles the critical point when $\delta_{p}=0$, and consequently in this case we have $\Delta\left(G_{s p}\right) \leq 0$. This last case is illustrated in Figure $5 \mathrm{~B}$.

For item $(i i)$, knowing that the two transfer functions $H_{s}$ and $H_{g}$ are input-output stable, and that $H_{g}$ is strictly decreasing, we have $H_{s g}$ is input-output stable if and only if $\delta_{g}<\Delta\left(G_{s g}\right)$, like in the case of item $(i)$. When $c_{g} \sigma_{g}^{\star} \sigma_{s}^{\star}<1+c_{g}^{g} \sigma_{g}^{\star}$ we have $\Delta\left(G_{s g}\right)=+\infty$. This case is illustrated in Figure $5 \mathrm{C}$. While, when $c_{g} \sigma_{g}^{\star} \sigma_{s}^{\star} \geq 1+c_{g}^{g} \sigma_{g}^{\star}$, the delay margin of the system is positive. This last case is illustrated in Figure $5 \mathrm{D}$. 
Proof of Theorem 2 We have that $H_{s p}$ and $H_{g}$ are input-output stable. If $\gamma_{H}$ is strictly decreasing then, from [18, Theorem 2], we have that the delayed feedback system $\left(H_{s p}, H_{g}\right)$ is input-output stable if and only if $\delta_{g}<\Delta(H)$. Moreover, by Nyquist Theorem, we know that the delayed feedback system $\left(H_{s p}, H_{g}\right)$ is input-output stable if and only if $\operatorname{ind}\left(1-H_{s p} K_{g}\right)=0$, which equivalent from Lemma 2 to $\operatorname{ind}\left(1-H_{s}\left(K_{p}+K_{g}\right)\right)=0$. Then, one can conclude with Proposition 5 that the equilibrium point is exponentially stable for (4) if and only if $\delta_{g}<\Delta(H)$.

Proof of Corollary 1 We know, from Lemma 3, that for each $\delta_{p}>0$ one can find $c_{p}^{\star}>0$ such that the gain $\gamma_{H_{s p}}$ is strictly decreasing for every $c_{p}$ in the interval $\left(0, c_{p}^{\star}\right)$. Moreover, from [18, Lemma 4$]$, one can find, for each set of parameters, a positif real number $\delta_{g}^{g \star}$ such that the gain $\gamma_{H_{g}}$ is strictly decreasing for every $\delta_{g}^{g}<\delta_{g}^{g \star}$. Therefore, for each $\delta_{p}>0$, if $\gamma_{H_{g}}$ is strictly decreasing, one can find $c_{p}^{\star}>0$ such that the gain $\gamma_{H}$ is strictly decreasing. Thus, in this case, Theorem 2 ends the proof of the corollary.

\section{A Frequency domain methods}

Suppose that $\beta$ is any real number. Let $\mathbb{C}_{\beta}^{+}$and $\overline{\mathbb{C}_{\beta}^{+}}$define the following two sets, respectively,

$$
\mathbb{C}_{\beta}^{+}:=\{s \in \mathbb{C}: \operatorname{Re}(s)>\beta\} \quad \text { and } \quad \overline{\mathbb{C}_{\beta}^{+}}:=\{s \in \mathbb{C}: \operatorname{Re}(s) \geq \beta\}
$$

Most of the concepts recalled here are taken from [5].

\section{A.1 Convolution algebras}

We say that $f \in \mathcal{A}(\beta)$ if it has the representation

$$
f(t)= \begin{cases}f_{a}(t)+\sum_{n=1}^{\infty} f_{n} \delta\left(t-t_{n}\right) & t \geq 0 \\ 0 & t<0\end{cases}
$$

where $e^{-\beta t} f_{a}(t) \in L_{1}(0, \infty), f_{n} \in \mathbb{C}, t_{n} \in \mathbb{R} ; t_{1}=0, t_{n}>0$ for $n \geq 2, \delta\left(t-t_{n}\right)$ represents the delta distribution centred in $t_{n}$ and $\sum_{n=1}^{\infty}\left|f_{n}\right| e^{-\beta t_{n}}<\infty$. See [5, Definition A.7.45, p. 661] for more details. Any function $f \in \mathcal{A}(\beta)$ possesses the Laplace transform $\hat{f}(\cdot)$ in $\mathbb{C}_{\beta}^{+}$given by:

$$
\hat{f}(s)=\int_{0}^{\infty} e^{-s t} f_{a}(t) d t+\sum_{n=1}^{\infty} f_{n} e^{-t_{n} s} \quad \forall s \in \mathbb{C}_{\beta}^{+} .
$$

Consider the class of Laplace transforms of $\mathcal{A}(\beta)$ given by:

$$
\hat{\mathcal{A}}(\beta):=\{\hat{f}: f \in \mathcal{A}(\beta)\} .
$$

The set $\hat{\mathcal{A}}(\beta)$ is a commutative Banach algebra under the norm

$$
\|\hat{f}\|:=\sup _{s \in \overline{\mathbb{C}}_{\beta}^{+}}|\hat{f}(s)| .
$$

See [5, Corollary A.7.48, p. 665] for more details.

Let us introduce the two following subsets of $\hat{\mathcal{A}}:=\hat{\mathcal{A}}(0)$ :

$$
\hat{\mathcal{A}}_{-}:=\{\hat{f}: \exists \epsilon>0, \hat{f} \in \hat{\mathcal{A}}(-\epsilon)\}
$$

and

$$
\hat{\mathcal{A}}_{\infty}:=\left\{\hat{f} \in \hat{\mathcal{A}}_{-}: \exists \rho>0, \inf _{s \in \overline{\mathbb{C}_{0}^{+}},|s| \geq \rho}|\hat{f}(s)|>0\right\}
$$

We note that in $[5$, Definition 7.1 .4$, p. 338$]$ these two sets correspond to $\hat{\mathcal{A}}_{-}(0)$ and $\hat{\mathcal{A}}_{\infty}(0)$, respectively. According to [5, Lemma 7.1.5, p. 338], both $\hat{\mathcal{A}}_{-}$and $\hat{\mathcal{A}}_{\infty}$ inherit several properties from $\hat{\mathcal{A}}$, in particular: 
$\left(p_{1}\right) \hat{\mathcal{A}}_{-}$is a subalgebra of $\hat{\mathcal{A}}$;

$\left(p_{2}\right)$ Any $\hat{f} \in \hat{\mathcal{A}}_{-}$is holomorphic and bounded on $\overline{\mathbb{C}_{0}^{+}}$;

$\left(p_{3}\right)$ Any $\hat{f} \in \hat{\mathcal{A}}_{-}$is invertible over $\hat{\mathcal{A}}_{-}$if and only if $\inf _{s \in \overline{\mathbb{C}}_{0}^{+}}|\hat{f}(s)|>0$.

From the definition of $\hat{\mathcal{A}}_{\infty}$, we know that for a given $\hat{f} \in \hat{\mathcal{A}}_{\infty}$, there exists a $\epsilon>0$ such that $\hat{f} \in \hat{\mathcal{A}}(-\epsilon)$. Consequently, from the properties of $\hat{\mathcal{A}}(-\epsilon), \hat{f}$ is holomorphic over $\mathbb{C}_{-\epsilon}^{+}$and so it has only finitely many isolated zeros of finite order in the compact subset $\left\{s \in \overline{\mathbb{C}_{0}^{+}}:|s| \leq \rho\right\}$ of $\mathbb{C}_{-\epsilon}^{+}[5$, Theorem A.1.4, p. 566]. By definition, it cannot have zeros in the subset $\left\{s \in \overline{\mathbb{C}_{0}^{+}}:|s| \geq \rho\right\}$. Then, an important property of elements belonging to $\hat{\mathcal{A}}_{\infty}$ is the following

$\left(p_{4}\right) \hat{f} \in \hat{\mathcal{A}}_{\infty}$ has only finitely many zeros of finite order in $\overline{\mathbb{C}_{0}^{+}}$.

\section{A.2 Nyquist's Theorem}

In this subsection we recall the definition of input-output stability of irrational transfer matrix and then we introduce the Nyquist Theorem in its general form. Firstly, it is necessary to define the class of scalar irrational transfer functions. The quotient algebra $\hat{\mathcal{B}}$ of $\hat{\mathcal{A}}_{-}$with respect to $\hat{\mathcal{A}}_{\infty}$ represents the class of scalar (possibly unstable) irrational transfer functions $\hat{\mathcal{B}}$, which is a particular case of the Callier-Desoer class [5, Definition 7.1.6], and that is given by

$$
\hat{\mathcal{B}}:=\left\{F=\hat{n} \hat{m}^{-1}: \hat{n} \in \hat{\mathcal{A}}_{-}, \hat{m} \in \hat{\mathcal{A}}_{\infty}\right\} .
$$

Observe that, obviously, $\hat{\mathcal{B}} \subset \hat{\mathcal{A}}_{-}$.

Let $\mathcal{M} \hat{\mathcal{A}}_{-}$and $\mathcal{M} \hat{\mathcal{B}}$ denote the set of matrices (of any size) with elements in $\hat{\mathcal{A}}_{-}$and $\hat{\mathcal{B}}$ respectively. Using the mathematical structure outlined above, the appropriate stability concepts for transfer matrices is defined by the following definition.

Definition 1 [5, Definition 9.1.1, p. 457] $G \in \mathcal{M \mathcal { B }}$ is said to be input-output stable if $G \in \mathcal{M} \hat{\mathcal{A}}_{-}$.

Furthermore, the connection between the input-output stability of the transfer matrix $G$ and the exponential stability of the semigroup generated by its infinite-dimensional state-space representation is given in [5, Theorem 7.3.2, p. 364].

Definition 2 [5, Definition 9.1.2, p. 458] A feedback system $(G, K)$, where $G, K \in \mathcal{M} \hat{\mathcal{B}}$, is said to be input-output stable if and only if

a. There exists a $\rho>0$ such that

$$
\inf _{s \in \mathbb{C}_{0}^{+},|s| \geq \rho}|\operatorname{det}(I-G(s) K(s))|>0
$$

b. The transfer matrices $S:=(I-G K)^{-1}, K S, S G$ and $I+K S G$ are in $\mathcal{M} \hat{\mathcal{A}}_{-}$.

We recall that two transfer matrices $M, N \in \mathcal{M} \hat{\mathcal{A}}_{-}$are right-coprime over $\mathcal{M} \hat{\mathcal{A}}_{-}$, if their have the same number of columns, and there exist $\tilde{X}, \tilde{Y} \in \mathcal{M} \hat{\mathcal{A}}_{-}$such that the following Bezout identity holds on $\overline{\mathbb{C}_{0}^{+}}$:

$$
\tilde{X} M-\tilde{Y} N=I .
$$

We refer to [5, Definition 7.2.6, p. 352] to the complete definition concerning the left- and right-coprime matrices.

The interesting consequence arising from the use of $\mathcal{M} \hat{\mathcal{B}}$, to define the class of irrational transfer functions, is that all elements of $\mathcal{M} \hat{\mathcal{B}}$ always possess both left- and right-coprime factorization over $\mathcal{M} \hat{\mathcal{A}}_{-}[5$, Theorem 7.2.8, p. 353]. In other words, for each matrix $G \in \mathcal{M B \mathcal { B }}$, there exist two transfer matrices $M, N \in \mathcal{M} \hat{\mathcal{A}}_{-}$such that $M$ and $N$ are left-coprime (right-coprime) over $\mathcal{M} \hat{\mathcal{A}}_{-}, N$ is a square matrix 
with $\operatorname{det}(N) \in \hat{\mathcal{A}}_{\infty}$ and $G=M N^{-1}\left(G=N^{-1} M\right)$. These factorizations are unique up to a common left (right) multiplication by an invertible element in $\mathcal{M} \hat{\mathcal{A}}_{-}$. Since $\operatorname{det}(N) \in \hat{\mathcal{A}}_{\infty}$, property $\left(p_{4}\right)$ guaranties that $\operatorname{det}(N)$ has only finitely many zeros of finite order in $\overline{\mathbb{C}_{0}^{+}}$. By definition [5, Definition 7.2.18, p. 359], the poles of $G$ in $\overline{\mathbb{C}_{0}^{+}}$are equal to the zeros of $\operatorname{det}(N)$ in $\overline{\mathbb{C}_{0}^{+}}$and their McMillan degree is defined as the order of the zeros.

Definition 3 [5, Definition 7.2.4, p. 351] $G \in \mathcal{M} \hat{\mathcal{B}}$ is said to be proper on $\overline{\mathbb{C}_{0}^{+}}$if for sufficiently large $\rho>0$,

$$
\sup _{s \in \mathbb{C}_{0}^{+},|s| \geq \rho}|G(s)|<\infty .
$$

$G \in \mathcal{M} \hat{\mathcal{B}}$ is said to be strictly proper on $\overline{\mathbb{C}_{0}^{+}}$if

$$
\lim _{\rho \rightarrow \infty}\left[\sup _{s \in \overline{\mathbb{C}_{0}^{+},|s| \geq \rho}}|G(s)|\right]=0 .
$$

$G \in \mathcal{M B}$ is said to have a limit at infinity in $\overline{\mathbb{C}_{0}^{+}}$if there exists a $G_{\infty} \in \mathcal{L}\left(\mathbb{C}^{m}, \mathbb{C}^{k}\right)$ such that

$$
\lim _{\rho \rightarrow \infty}\left[\sup _{s \in \overline{\mathbb{C}_{0}^{+}},|s| \geq \rho}\left|G(s)-G_{\infty}\right|\right]=0 .
$$

$G_{\infty}$ is called the limit at infinity.

Theorem 3 Nyquist Theorem. [5, Theorem 9.1.8, p. 463] Suppose that $G, K \in \mathcal{M} \hat{\mathcal{B}}$ have $p_{G}$ and $p_{K}$ poles, respectively, in $\mathbb{C}_{0}^{+}$, counted according to their McMillan degree. If $G K$ is strictly proper on $\overline{\mathbb{C}_{0}^{+}}$, then the feedback $(G, K)$ is input-output stable if and only if

$$
\operatorname{ind}(\operatorname{det}(I-G K))=-p_{K}-p_{G},
$$

where $\operatorname{ind}(\operatorname{det}(I-G K))$ denotes the Nyquist index [5, Definition A.1.15, p. 569] of $\operatorname{det}(I-G K)$.

Corollary 2 Nyquist Test. [5, Corollary 9.1.9, p. 465] Suppose that $G, K \in \mathcal{M} \hat{\mathcal{B}}$ have $p_{G}$ and $p_{K}$ poles, respectively, in $\mathbb{C}_{0}^{+}$, counted according to their McMillan degree and neither poles nor zeros on the imaginary axis. If $G K$ is strictly proper on $\overline{\mathbb{C}_{0}^{+}}$, then the feedback $(G, K)$ is input-output stable if the plot of $\operatorname{det}(I-G K)(i w)$ as $w$ decreases from $\infty$ to $-\infty$ encircles the origin $\left(p_{G}+p_{K}\right)$ times in the clockwise sense.

\section{References}

[1] R. Ammari and B. Bioulac and L. Garcia and C. Hammond, The subthalamic nucleus becomes a generator of bursts in the dopamine-depleted state. Its high frequency stimulation dramatically weakens transmission to the globus pallidus, Frontiers in Systems Neuroscience, vol. 5, 2011.

[2] M.D. Bevan and J.P. Bolam, Cholinergic, GABAergic, and glutamate-enriched inputs from the mesopontine tegmentum to the subthalamic nucleus in the rat, J Neurosci., vol. 15, no. 11, pp. 7105-7120, Nov. 1995.

[3] T. Boraud, P. Brown, J.A. Goldberg, A.M. Graybiel and P.J. Magill, Oscillations in the basal ganglia: the good, the bad, and the unexpected, Advances in behavioral biology (The Basal Ganglia VIII, Editors: Bolam, J.P., Ingham, C.A. and Magill, P.J.), vol. 56, pp. 124, 2005.

[4] C. Martinez-Gonzalez, J. Paul-Bolam and J. Mena-Segovia, Topographical organization of the pedunculopontine nucleus, Front Neuroanat., vol. 5, no. 22, Apr. 2011. 
[5] R.F. Curtain and H. Zwart, An introduction to infinite-dimensional linear systems theory, SpringerVerlag, vol. 21, Apr. 1995.

[6] P. Dayan and L.F. Abbott, Computational and mathematical modelling of neural systems, Theoretical neuroscience, MIT Press, 2001.

[7] A.R. Granata and S.T. Kitai, Intracellular analysis of excitatory subthalamic inputs to the pedunculopontine neurons, Brain Res., vol. 488, pp. 57-72, May 1989.

[8] C. Hamani, J.A. Saint-Cyr, J. Fraser, M. Kaplitt and A.M. Lozano, The subthalamic nucleus in the context of movement disorders, Brain, vol. 127, pp. 4-20, Jan. 2004

[9] C. Hammond, B. Rouzaire-Dubois, J. Feger, A. Jackson, and A.R. Crossman, Anatomical and electrophysiological studies on the reciprocal projections between the subthalamic nucleus and nucleus tegmenti pedunculopontinus in the rat, Neuroscience, vol. 9, no. 1, pp. 41-52, 1983.

[10] A.L. Nevado-Holgado, J.R. Terry and R. Bogacz, Conditions for the generation of beta oscillations in the subthalamic nucleus-globus pallidus network, The Journal of Neuroscience, vol. 30, no. 37, pp. 12340-12352, Sep. 2010.

[11] H.K. Khalil, Nonlinear systems, Prentice Hall, 2002.

[12] H. Kita, Globus pallidus external segment, Prog Brain Res., vol. 160, pp. 111-133, 2007.

[13] A. Leblois, T. Boraud, W. Meissner, H. Bergman and D. Hansel, Competition between Feedback Loops Underlies Normal and Pathological Dynamics in the Basal Ganglia, The Journal of Neuroscience, vol. 26, no. 13, pp. 3567-3583, Mar. 2006.

[14] J. Liu, K.G. Oweiss and H.K. Khalil, Feedback control of the spatiotemporal firing patterns of neural microcircuits, 49th IEEE Conference on Decision and Control, December 2010.

[15] M.A.J. Lourens, H.G.E. Meijer, T. Heida, E. Maranib and S.A. van Gils, The pedunculopontine nucleus as an additional target for deep brain stimulation, Neural Networks, vol. 24, no. 6, pp. 617-630, 2011.

[16] N.M McCarthy, C. Moore-Kochlacs, X. Gu, E.S. Boyden, X. Han and N. Kopell, Striatal origin of the pathologic beta oscillations in Parkinson's disease, Proc. of the National Academy of Sciences, vol. 108, no. 28, pp. 11620-11625, 2011.

[17] J.A. Obeso and J.L. Lanciego, Past, present, and future of the pathophysiological model of the basal ganglia, Front Neuroanat., vol. 5, no. 39, Jul. 2011.

[18] W. Pasillas-Lépine, Equilibrium and stability of Wilson and Cowan's time coarse graining model, Proceedings of MTNS 2012-Mathematical Theory of Networks and Systems, http://hal.archivesouvertes.fr/hal-00692085

[19] A. Pavlides, S.J. Hogan and R. Bogacz, Improved conditions for the generation of beta oscillations in the subthalamic nucleus-globus pallidus network, European Journal of Neuroscience, vol. 36, pp. 2229-2239, 2012.

[20] D. Plenz and S.T. Kital, A basal ganglia pacemaker formed by the subthalamic nucleus and external globus pallidus, Nature, vol. 400 n0. 6745, pp. 677682, Aug. 1999.

[21] J. Mena-Segovia, J.P. Bolam and P.J. Magill, Pedunculopontine nucleus and basal ganglia: distant relatives or part of the same family?, Trends in Neurosciences, vol. 27, no. 10, pp. 585-588, Oct. 2004.

[22] D. Terman and J.E. Rubin and A.C. Yew and C.J. Wilson, Activity patterns in a model for the subthalamopallidal network of the basal ganglia, The Journal of neuroscience, vol. 22, no. 7, pp. 2963-2976, 2002. 
[23] J.R. Walters, D. Hu, C.A. Itoga, L.C. Parr-Brownlie, D.A. Bergstrom, Phase relationships support a role for coordinated activity in the indirect pathway in organizing slow oscillations in basal ganglia output after loss of dopamine, Neuroscience, vol. 144, no. 2, pp. 762776, Jan. 2007

[24] H.R.Wilson and J.D. Cowan, Excitatory and inhibitory interactions in localized populations of model neurons, Biophysical journal, vol. 12, no. 1, pp. 1-24, 1972

[25] N. Yamawaki and I.M. Stanford and S.D. Hall and G.L. Woodhall, Pharmacologically induced and stimulus evoked rhythmic neuronal oscillatory activity in the primary motor cortex in vitro, Neuroscience, vol. 151, no. 2, pp. 386-395, 2008. 